\title{
Phase behaviour and gravity-directed self assembly of hard convex spherical caps
}

DOI:

10.1039/C6SM02678H

Document Version

Accepted author manuscript

Link to publication record in Manchester Research Explorer

\section{Citation for published version (APA):}

Mcbride, J., \& Avendano, C. (2017). Phase behaviour and gravity-directed self assembly of hard convex spherical caps. Soft Matter. https://doi.org/10.1039/C6SM02678H

\section{Published in:}

Soft Matter

\section{Citing this paper}

Please note that where the full-text provided on Manchester Research Explorer is the Author Accepted Manuscript or Proof version this may differ from the final Published version. If citing, it is advised that you check and use the publisher's definitive version.

\section{General rights}

Copyright and moral rights for the publications made accessible in the Research Explorer are retained by the authors and/or other copyright owners and it is a condition of accessing publications that users recognise and abide by the legal requirements associated with these rights.

\section{Takedown policy}

If you believe that this document breaches copyright please refer to the University of Manchester's Takedown Procedures [http://man.ac.uk/04Y6Bo] or contact uml.scholarlycommunications@manchester.ac.uk providing relevant details, so we can investigate your claim.

\section{OPEN ACCESS}




\title{
Journal Name
}

\section{ARTICLE TYPE}

Cite this: DOI: $10.1039 / x x x x x x x x x x$

\section{Phase behaviour and gravity-directed self assembly of hard convex spherical caps}

\author{
John M. McBride and Carlos Avendaño*
}

Received Date

Accepted Date

DOI: $10.1039 / x x x x x x x x x x$

www.rsc.org/journalname
We investigate the phase behaviour and self-assembly of convex spherical caps using Monte Carlo simulations. This model is used to represent the main features observed in experimental colloidal particles with mushroom-cap shape [Riley et al., Langmuir, 2010, 26, 1648]. The geometry of this non-centrosymmetric convex model is fully characterized by the aspect ratio $\chi^{*}$ defined as the spherical cap height to diameter ratio. We use NPT Monte Carlo simulations combined with free energy calculations to determine the most stable crystal structures and the phase behaviour of convex spherical caps with different aspect ratios. We find a variety of crystal structures at each aspect ratio, including plastic and dimer-based crystals; small differences in chemical potential between the structures with similar morphology suggest that convex spherical caps have the tendency to form polycrystalline phases rather than crystallising into a single uniform structure. With the exception of plastic crystals observed at large aspect ratios $\left(\chi^{*}>0.75\right)$, crystallisation kinetics seem to be too slow, hindering the spontaneous formation of ordered structures. As an alternative, we also present a study of directing the self-assembly of convex spherical caps via sedimentation onto solid substrates. This study contributes to show how small changes to particle shape can significantly alter the self-assembly of crystal structures, and how a simple gravity field and a template can substantially enhance the process.

\section{Introduction}

Self-assembly of molecular, nano, and colloidal building blocks is an efficient method of producing functional materials. ${ }^{1,2}$ This method has become particularly important for the formation of materials across the colloidal length scale due to recent developments of experimental techniques to synthesise particles with arbitrary shape and surface functionalities. ${ }^{3-8}$ The goal of selfassembly is to understand the role of shape and interparticle interactions on the spatial arrangement of a system, leading to formulation of design rules to target specific structures by controlling the driving forces affecting the organisation of the building blocks. 9,10 The role of shape alone has been extensively explored by considering particles that interact purely via repulsive interactions. ${ }^{11-22}$

Computer simulations of hard-core particle models have played a significant role in understanding the mechanism behind the organisation of colloidal systems. A variety of shapes in bulk and under the influence of external fields have been explored including spherocylinders ${ }^{15-18}$, cut-spheres ${ }^{23}$ and

${ }^{a}$ School of Chemical Engineering and Analytical Science, The University of Manchester, Sackville Street, Manchester M13 9PL, UK. Tel: +44 (0)161 306 2732; E-mail: carlos.avendano@manchester.ac.uk platelets $^{24-28}$, dimers ${ }^{29-31}$, bowl-like particles ${ }^{32-34}$, concave spherical caps ${ }^{35-37}$, polyhedra ${ }^{38-42}$, and a variety of branched particles ${ }^{43-46}$, just to mention few examples. An assumption behind these studies is that the effect of gravitational forces is negligible, corresponding to a situation of colloidal particles embedded within a density matched (implicit) medium. Away from these conditions, however, the effect of gravity can no longer be ignored 47 . The influence of gravity on a suspension is typically measured in terms of the relative strength between thermal energy due to Brownian motion and the gravitational energy. When gravitational effects become important, density inhomogeneities develop along the direction of the field resulting in sedimentation and even segregation and phase transformations ${ }^{48-54}$. In some cases, gravitational effects can be used to program selfassembly $^{9,55}$.

In this work, we present a computer simulation study of a model of convex spherical caps ${ }^{56}$ in bulk and under a gravitational field to direct their self-assembly. The particle model, shown in Figure 1, is used to represent the features of real mushroom cap-shaped (MCS) colloidal particles ${ }^{57-61}$. Under strong geometrical confinement, MCS particles exhibit a very rich phase behaviour due to their anisotropic shape and lack of centrosymmetry ${ }^{58,62}$. This behaviour has been confirmed by com- 
puter simulations of a convex spherical cap model, demonstrating the suitability of the model ${ }^{56}$. Moreover, MCS particles have gained considerable attention due to the possibility of forming photonic band-gap materials with highly anisotropic Brillouin zones. ${ }^{58,63-65}$ The focus of the present report is to use Monte Carlos (MC) computer simulations to explore the competing effects of gravity and particle shape on crystal structures formed on both planar structureless walls and patterned substrates to study colloidal epitaxy ${ }^{66}$. A good understanding of crystallisation by sedimentation is necessary if it is to be a viable route for the production of novel structures from anisotropic particles.

\section{Methods}

The convex spherical cap (CSC) model used in this work consists of a hard sphere of diameter $\sigma$ cut off by a plane at a height $\chi$, as shown in Figure 1. The geometry of the particle is described by its aspect ratio $\chi^{*}=\chi / \sigma$. The algorithm to detect overlaps between particles has been presented elsewhere. ${ }^{32,56,67}$ We first analyse the formation of ordered structures of CSCs with different aspect ratios in bulk conditions, followed by the analysis of the structures formed under the influence of a gravitational field. Properties are reported in dimensionless units: pressure, $P^{*}=P \sigma^{3} /\left(k_{B} T\right)$, density, $\rho^{*}=N \sigma^{3} / V$, and volume fraction, $\phi=N v_{p} / V$, where $P$ is the pressure, $k_{B}$ is the Boltzmann constant, $T$ is the absolute temperature, $N$ is the total number of particles, $v_{p}=\pi \chi^{2}(3 \sigma / 2-$ $\chi) / 3$ is the volume of a CSC of height $\chi$, and $V$ is the total volume of the system. Similarly, all lengths are expressed in units of $\sigma$.

\subsection{Bulk simulations}

The search for bulk equilibrium structures is broken up into two stages. First we determine the most stable crystal structures for each aspect ratio, followed by the analysis of the regions of stability of the structures. Free energy calculations are used to determine these regions of stability. For crystal phases the free energy is determined using the Einstein crystal method. ${ }^{32,68-70}$ Details of the implementation of this method are given in the Appendix. For the fluid phase the excess chemical potential is obtained using Widom's particle insertion method ${ }^{71}$. Close-packed crystal structures are determined using the floppy-box MC algorithm, hereafter referred to as FBMC simulations. ${ }^{72-74}$ This method entails generating several hundred candidate crystal structures for each

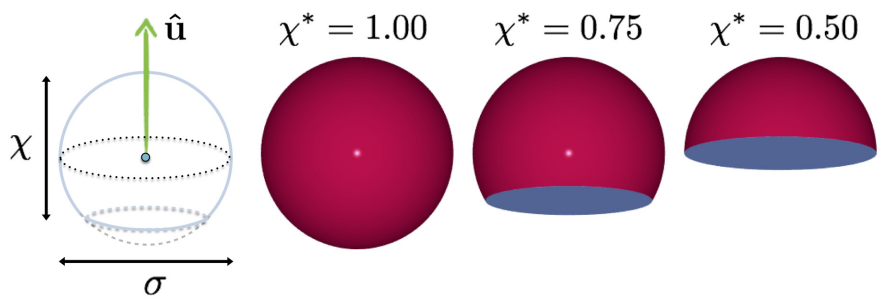

Fig. 1 Representation of the geometry of the hard-core convex spherical caps model. The geometry of the model is uniquely defined by the aspect ratio $\chi^{*}=\chi / \sigma$, where $\chi$ is the height of the cap and $\sigma$ is the diameter of the parental sphere. The model has axial symmetry and its orientation is described by the unit vector $\hat{\mathbf{u}}$. Note that the value of $\chi^{*}=1$ corresponds to a sphere, while $\chi^{*}=0.5$ corresponds to a hemisphere. aspect ratio and selecting unique candidates as possible equilibrium structures. For each aspect ratio $\chi^{*}$, and each number of particles from 2 to 10, one hundred configurations are generated using different initial conditions. Each system is compressed from a pressure of $P^{*}=4$ to $P^{*}=20$ in steps of $\Delta P^{*}=4$, then to a pressure of $P^{*}=100$ in steps of $\Delta P^{*}=20$, and finally to a pressure of $P^{*}=10^{6}$ by increasing the pressure by factors of 10 . Simulations are run in the isobaric-isotension NPT ensemble where the shape and size of the box are allowed to vary; $10^{6} \mathrm{MC}$ cycles are performed at each pressure. Periodic boundary conditions are applied in all directions. A cycle consists of $N$ MC moves with the following probabilities: translation, $30 \%$; rotation $30 \%$; $180^{\circ}$ rotation, $10 \%$; isotropic volume change $15 \%$; anisotropic volume change $15 \%$. Moves are accepted based on the Metropolis algorithm and for the equilibration stage the acceptance rate is kept at about $30 \%$. Each final configuration is saved for post-processing analysis.

Crystal structures are analysed using translational and orientational order parameters. The crystal symmetry is determined using Steinhardt bond-orientational order parameters, $Q_{l}$, for $l=4$ and $l=6 .{ }^{75,76}$ The global orientational order of the system is characterised by computing the eigenvalues of the orientational tensor $\mathbf{Q}$ given in terms of the orientations of the particles $\hat{\mathbf{u}}_{i}$ as

$$
\mathbf{Q}=\frac{1}{N} \sum_{i=1}^{N} \frac{3}{2} \hat{\mathbf{u}}_{i} \otimes \hat{\mathbf{u}}_{i}-\frac{\mathbf{I}}{2},
$$

where I is the unit tensor. The director $\hat{\mathbf{u}}_{\text {sys }}$, i.e., the principal orientation of the system, corresponds to the eigenvector associated to the largest eigenvalue $S_{2}$ of the tensor $\mathbf{Q}$. $S_{2}$, usually referred to as the nematic order parameter ${ }^{77}$, is a measure of alignment along the principle axis of the system with $S_{2}=0$ indicating an isotropic system and $S_{2}=1$ indicating perfect alignment. $S_{2}$, however, does not differentiate between parallel or antiparallel orientations along the director. The orientational order parameter $S_{1}$ is useful for finding the proportion of particles aligned in the same direction and it is given by

$$
S_{1}=\frac{1}{N} \sum_{i=1}^{N} \hat{\mathbf{u}}_{i} \cdot \hat{\mathbf{u}}_{\text {sys }} .
$$

Given the propensity of CSCs to dimerise via contacts between their flat faces, the fraction of particles that form dimers is another indicator of crystal structure. Dimers are identified as having a small cap-to-cap distance between the centres of the CSCs faces and opposing orientations, $\hat{\mathbf{u}}_{i}$ and $\hat{\mathbf{u}}_{j}$. Two particles $i$ and $j$ form a dimer if the distance between their flat caps is $\left|\mathbf{r}_{i j}^{\mathrm{cap}}\right|<0.25$, with

$$
\mathbf{r}_{i j}^{\mathrm{cap}}=\mathbf{r}_{i j}^{\mathrm{com}}-\left[\chi-\sigma / 2+\frac{3(\sigma-\chi)^{2}}{4(3 \sigma / 2-\chi)}\right]\left(\hat{\mathbf{u}}_{j}-\hat{\mathbf{u}}_{i}\right),
$$

where $\mathbf{r}_{i j}^{\text {com }}$ is the vector between the centres of mass of particles $i$ and $j$.

Crystal structures with unique properties for each value of $\chi^{*}$ are then used as starting configurations for expansion runs in the isobaric-isotension NPT ensemble to determine the $\phi-P^{*}$ equation of state. Systems of approximately $N=500$ particles are ex- 
panded from the limit of close packing all the way to dilute gas. Periodic boundary conditions are applied in the three Cartesian coordinates. For each thermodynamic state, $10^{5} \mathrm{MC}$ cycles are performed for both equilibrium runs and for final ensemble averages. A cycle consists of $N$ MC moves of type and probability: translation, $40 \%$; rotation $40 \%$; $180^{\circ}$ rotation, $10 \%$; isotropic volume change 5\%; anisotropic volume change 5\%. Only isotropic volume changes are used for fluid states to avoid excessively deformed simulation cells. Phase coexistence points along the equation of state are determined by finding the point at which the chemical potential and pressure of two phases are equal.

It is useful to measure the degree of mobility of the particles. For this purpose, we calculate both the pseudo mean square displacement (MSD) $\Delta \mathbf{r}^{2}$ and the pseudo orientation autocorrelation function $E_{2}$ as

$$
\left\langle\Delta \mathbf{r}^{2}\left(t_{\mathrm{MC}}\right)\right\rangle=\left\langle\frac{1}{N} \sum_{i=1}^{N}\left[\mathbf{r}_{i}\left(t_{\mathrm{MC}}\right)-\mathbf{r}_{i}(0)\right]^{2}\right\rangle
$$

and

$$
E_{2}\left(t_{\mathrm{MC}}\right)=\left\langle\frac{1}{N} \sum_{i=1}^{N} \frac{1}{2}\left\{3\left[\hat{\mathbf{u}}_{i}(0) \cdot \hat{\mathbf{u}}_{i}\left(t_{\mathrm{MC}}\right)\right]^{2}-1\right\}\right\rangle
$$

where $t_{\mathrm{MC}}$ denotes Monte Carlo cycles. These are calculated using NVT-MC simulations with fixed values for the maximum particle displacement and rotation ${ }^{78}$.

\subsection{Simulations of convex spherical caps under gravity}

The behaviour of CSCs under the influence of a gravitational field is studied using $N V T$-MC simulations. Particles are placed in a simulation box cell of dimensions $L_{x}, L_{y}$, and $L_{z}$. Periodic boundary conditions are applied only in the $x$ and $y$ directions, while a hard wall is placed at the bottom of the cell along the $z$ direction. This situation corresponds to a finite system where particles are confined within a large slit pore of volume $V=L_{x} L_{y} L_{z}$. The particles are subject to an external field of the form

$$
\phi_{i}(z)=m g z_{i}
$$

where $z_{i}$ is the $z$-component of the centre of mass of particle $i, m$ is the buoyant mass, and $g$ is the gravitational acceleration. The origin of the cell is located in the middle of the box. The algorithm to detect overlaps between a CSCs and a hard wall is taken from reference [56]. The strength of the gravity field is controlled by the gravitational length given by $l_{g}^{*}=l / \sigma=k_{B} T / m g \sigma$. Note that the lower the value of $l_{g}^{*}$ is, the stronger the gravitational field is with respect to the thermal energy.

The box area $A=L_{x} L_{y}$ is adjusted to accommodate a hexagonal arrangement of spheres of diameter $\sigma .50$ This is achieved by setting $L_{x}=n a_{0}, L_{y}=\frac{\sqrt{3}}{2} n a_{0}$, where $n$ is an integer and $a_{0}$ is the lattice constant. While the choice of $a_{0}$ has a non-negligible effect on the crystallisation of the particles, crystal strain due to lattice-mismatch is still unavoidable as the shape and size of the box are kept constant while the system undergoes an effective compression. ${ }^{50,79}$ An intermediate value of $a_{0}=1.06$ is used in this work for all the simulations to accomodate crystals across a range of densities. Different values of particles per unit of area $\rho_{A}^{*}=N \sigma^{2} / A$ are used in the range of $2.5 \leq \rho_{A}^{*} \leq 25$. The number of particles $N$ used range from 1000 to 5000. For the majority of simulations the box is bounded at the bottom by a flat hard wall. However, additional simulations are performed where the box is bounded by a template constructed of monolayers of spheres frozen in place and arranged with hexagonal symmetry and lattice constant $a_{0}=1.06$.

MC-NVT simulation runs are started with random initial configurations at a high value of $l_{g}^{*}$, corresponding to a weak gravitational strength. The value of $l_{g}^{*}$ is then decreased sequentially, using as a starting configuration the previous final configuration at higher gravitational strength. For each value of $l_{g}^{*}, \sim 10^{6} \mathrm{MC}$ cycles are used for the equilibration stage, and additional $\sim 10^{6} \mathrm{MC}$ cycles are used to collect ensemble averages. Each cycle attempts $N$ Monte Carlo moves with the following probabilities: translation, $40 \%$; rotation, $40 \%$; $180^{\circ}$ rotation, $20 \%$. Moves are accepted using the Metropolis algorithm and the acceptance rate is tuned during the equilibration stage to an approximate value of $30 \%$.

The particle distribution along the $z$-direction is analysed by the area density profile $\rho_{A}^{*}(z)$,

$$
\rho_{A}^{*}(z)=\frac{1}{A} \iint \rho^{*}(x, y, z) \mathrm{d} x \mathrm{~d} y
$$

where $\rho^{*}(x, y, z)$ is the local reduced number density. Density profiles are calculated using bins of thickness $\Delta z=0.1 \sigma$. Maxima and minima in the density profile indicate the formation of layers and are used to isolate particles in well-defined layers for analysis of local translational and orientational order. Structural order along individual layers is analysed using two-dimensional bond-orientational order parameters $\Psi_{n}(z)$ along the $z$-direction defined as

$$
\Psi_{n}(z)=\frac{1}{N(z)} \sum_{j=1}^{N(z)} \frac{1}{N_{j}} \sum_{k=1}^{N_{j}} \exp \left(i n \theta_{j k}\right)
$$

where $n$ describes the degree of symmetry analysed, $N(z)$ is the number of particles in a layer at positions $z, N_{j}$ is the number of nearest neighbours of particle $j, \theta_{j k}$ is the angle made by the bond between particle $j$ and its nearest neighbour $k$ with respect to an arbitrary axis ${ }^{80}$. Neighbouring particles are determined using a Voronoi tessellation algorithm ${ }^{81}$. Local orientation of the particles is analysed using profiles of the orientational order parameter $S_{1}(z)$ and $S_{2}(z)$. The reduced osmotic pressure $P^{*}\left(z_{0}\right)$ is calculated as the force per unit of area exerted on a layer of particles at height $z_{0}$ by the overhead particles at height $z>z_{0}{ }^{50,82}$,

$$
P^{*}\left(z_{0}\right)=\frac{P\left(z_{0}\right) \sigma^{3}}{k T}=\frac{1}{\sigma A l_{g}^{*}} \iiint_{z_{0}}^{\frac{L_{z}}{2}} \rho^{*}(x, y, z) \mathrm{d} x \mathrm{~d} y \mathrm{~d} z .
$$

\section{Results}

\subsection{Bulk phase behaviour of convex spherical caps}

The phase behaviour of CSCs in bulk as a function of the aspect ratio $\chi^{*}$ has been analysed using NPT-MC simulations combined with free energy calculations. Figure 2 shows the stable crystal structures determined using FBMC simulations and free en- 
ergy calculations. These complex structures can be classified in 5 groups: isotropic disordered state $(\mathcal{I}$, not shown), plastic crystals $(\mathcal{P C})$, aligned crystals $(\mathcal{A})$, dimer-based crystals $(\mathcal{B})$, and interdigitated crystals $(\mathcal{C})$. The orientationally ordered crystals are further denoted by 1 or 2 depending on whether the CSCs are oriented along 1 or 2 axes.

It is not immediately obvious which crystal structure is the most stable at each value of aspect ratio $\chi^{*}$. While one can distinguish clearly between $\mathcal{P} C, \mathcal{A}, \mathcal{B}$, and $\mathcal{C}$ phases as they have different structural and thermodynamic properties, it is more difficult to differentiate between $\mathcal{A} 1$ and $\mathcal{A} 2$ crystals as they have similar properties. Furthermore, this is true for all other corresponding phases of the same crystal type $\left(\mathcal{B} 1_{P}\right.$ and $\mathcal{B} 2_{P}$, etc.). This is evident in Figure 3 where we show the maximum packing, $\phi_{m}$, obtained using FBMC simulations. As an example, $\mathcal{A} 1$ and $\mathcal{A} 2$ have essentially the same values of $\phi_{m}$ as a function of aspect ratio, in addition to having almost identical equations of state. It is interesting to note than although one would expect hemispheres $\left(\chi^{*}=0.5\right)$ to crystallize with a maximum value of $\phi_{m}=0.74$ in the $\mathcal{B}_{P C}$ crystal, these particles exhibit higher volume fractions since particles forming a dimer can experience small offsets to enhance the packing. This is also true for other $\mathcal{B}$ crystals for CSC with $\chi^{*}<0.65$ as observed in Figure 3. The structures shown in Figure 2 are in equilibrium, however the FCMB method is most apt for finding the densest crystal structures, and in the case of $\mathcal{B}$ crystals for $\chi^{*}<0.65$ the FBMC method finds structures which differ slightly from those reported in Figure 2. Even though the $\mathcal{P} C, \mathcal{A}$, $\mathcal{B}$, and $\mathcal{C}$ phases are distinct their stability needs to be assessed. It emerges that the relative stability of crystals must be determined by the interplay of translational and rotational entropy. One can provide an initial estimate of the stability of crystals by only considering the structure: high $\phi_{m}$ indicates high translational entropy; the rotational entropy may be inferred from a particle's local environment. So we first examine the structure of the different crystal phases shown in Figure 2.

The plastic crystal $\mathcal{P} C$ is characterised by particles arranged on a crystal lattice with random particle orientations. The $\mathcal{P} C$ phase for CSCs is found to be similar to the hard sphere crystal and the $F C C$ structure is used throughout this work. The $H C P$ structure is likely to be just as stable but the question of which is more stable is left for future work. In regions of stability $\mathcal{P} C$ is completely free to rotate ( $S_{2}$ is low at all volume fractions), which leads us to use linear scaling to determine the theoretical maximum packing in Figure 3. If one assumes that the unit cells of a CSC $\mathcal{P} C$ and a hard sphere $F C C$ crystal are identical at constant $\rho^{*}$, then the theoretical maximum packing is found as $\phi_{m}^{\mathcal{P} C}=\phi_{m}^{H S} v_{p} / v_{H S}$, where close packing of hard spheres is $\phi_{m}^{H S}=0.74$, and $v_{H S}$ is the volume of a sphere of diameter $\sigma$. It is evident that as $\chi^{*}$ decreases the maximum packing sharply drops while the rotational freedom is assumed unchanged, so the $\mathcal{P} C$ stability should be at a maximum at $\chi^{*}=1$ and decrease as a function of anisotropy. Crystals of type $\mathcal{A}$ have a deformed $F C C$ structure with a maximum $\phi_{m}$ at $\chi^{*}=0.85$. Both forms $\mathcal{A} 1$ and $\mathcal{A} 2$ exhibit almost identical packing and equations of state. The maximum packing of $\mathcal{A}$ crystals is always exceeded by that of the $\mathcal{B}$ crystals. Particles in $\mathcal{B}$ crystals are individually hindered from rotating due to their arrangement in
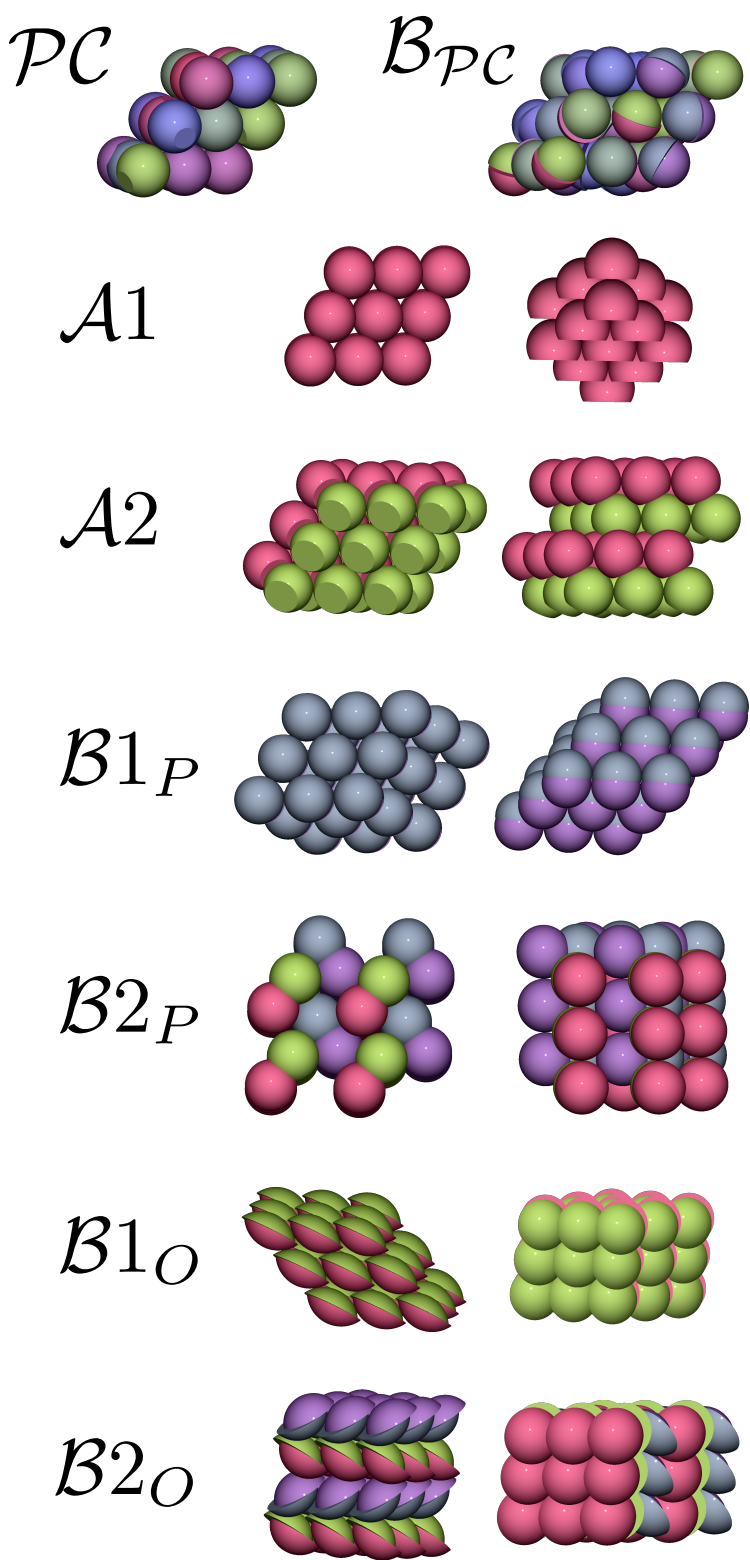

$\mathcal{C} 1$
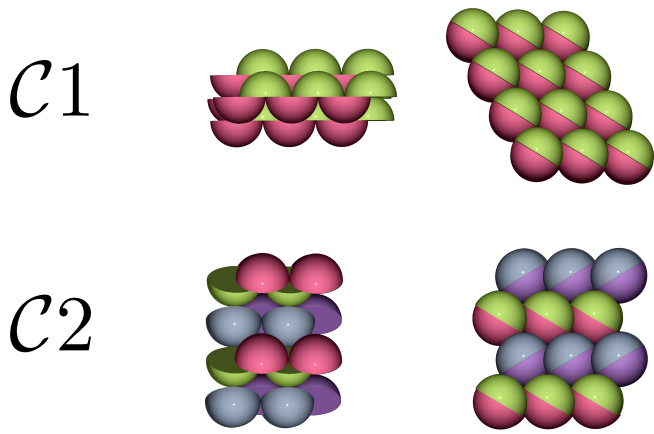

Fig. 2 Representative configurations of the ordered structures formed by CSCs with different values of aspect ratio $\chi^{*}: \mathcal{P C}, \chi^{*}=0.95 ; \mathcal{B}_{P C}$, $\chi^{*}=0.5 ; \mathcal{A} 1, \chi^{*}=0.75 ; \mathcal{A} 2, \chi^{*}=0.85 ; \mathcal{B} 1_{P}, \chi^{*}=0.6 ; \mathcal{B} 2_{P}, \chi^{*}=0.85$; $\mathcal{B} 1_{O}, \chi^{*}=0.25 ; \mathcal{B} 2_{O}, \chi^{*}=0.33 ; \mathcal{C} 1, \chi^{*}=0.5 ; \mathcal{C} 2, \chi^{*}=0.5$; The crystal structures are obtained using FBMC simulations. 


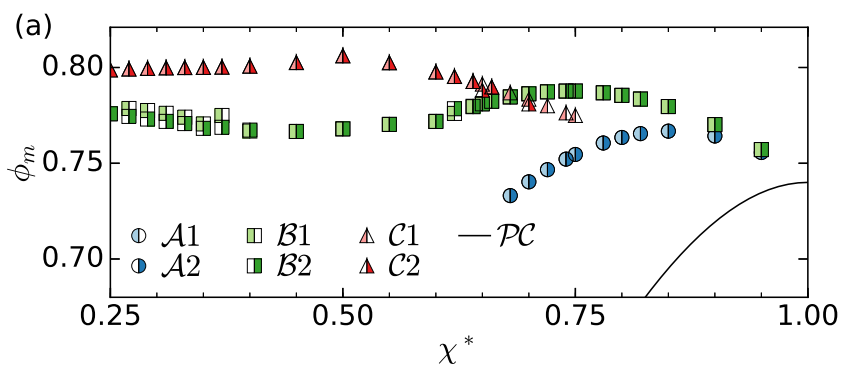

Fig. 3 Maximum volume fraction, $\phi_{m}$, obtained from FBMC simulations at various aspect ratios for crystals outlined in Figure 2. Theoretical maximum packing for $\mathcal{P C}$ crystals is given by the continuous curve.

dimer-like configurations. The tendency for CSCs to form these cap-to-cap contacts has been quantified by van Anders et al. ${ }^{83}$, and these arrangements are here referred to as specific entropic bonds (SEB). The most dense packings of CSCs always have at least one SEB as particles aligning cap-to-cap allows for more efficient use of space. Like with the $\mathcal{A}$ crystals, $\mathcal{B} 1$ and $\mathcal{B} 2$ have almost identical $\phi_{m}$ and equations of state. The $\mathcal{B}$ crystals however have more complex structures, and as $\chi^{*}$ decreases their structural properties change considerably. Crystals formed by CSCs of $\chi^{*} \gtrsim 0.6$ are denoted $\mathcal{B} 1_{P}$ and $\mathcal{B} 2_{P}$ as the dimers resemble prolate spheroids or dumbbells. Such a comparison draws on similarities between the $\mathcal{B} 2_{P}$ crystal and the structures observed in for hard ellipsoids by Dovev et al. ${ }^{84}$ and by Pfleiderer et al. ${ }^{85}$, and although it has not been studied here there may exist another $\mathcal{B}_{P}$ crystal where dimer bonds are formed randomly akin to the aperiodic crystal structure formed by dumbbells $29,31,86,87$. The next dimer-based crystal is $\mathcal{B}_{\mathcal{P C}}$ has similar structure to $\mathcal{P C}$ where in this case particles forming dimers can rotate cooperatively. First reported in Marechal et al. ${ }^{33}$ as $F C C^{2}$, this phase is an unusual example of a plastic crystal where the centres of mass of individual particles do not exhibit long-range translational order, as pairs of particles effectively orbit around the $F C C$ lattice points; longrange translational order is recovered if the particle reference is taken as the centre of mass of the dimers. Crystals formed by CSCs of $\chi^{*} \lesssim 0.4$ are denoted $\mathcal{B} 1_{O}$ and $\mathcal{B} 2_{O}$ as the dimers resemble oblate spheroids or UFO particles ${ }^{88}$, and are closely related to the structures observed in convex lens-shaped particles (note that a dimer formed by two CSCs have the same shape as a convex lens-shaped particles provided that $\left.0<\chi^{*} \leq 0.5\right)^{89}$. While the rotational freedom of individual particles is severely limited in $\mathcal{B}$ crystals, dimers can still rotate to varying degrees. This is in contrast to the $\mathcal{C}$ crystals where particles interdigitate and are effectively locked together. For $\mathcal{C}$ crystals the particles form two SEBs in order to achieve the most dense CSC packing, with a maximum $\phi_{m}$ located at $\chi^{*}=0.5$. Once again, $\mathcal{C} 1$ and $\mathcal{C} 2$ have almost identical $\phi_{m}$ and equations of state. The $\mathcal{C} 2$ structure can be considered $\mathrm{AB}$ stacking of layers with different orientations. Though not investigated here, a crystal with random stacking of layers may actually be the most stable $\mathcal{C}$ crystal.

Having described qualitatively the features of the different crystals we proceed to analyse their relative stability using free energy calculations. The results for the free energies of different crystal

\begin{tabular}{lccccc}
\hline$\chi^{*}$ & $P^{*}=P \sigma^{3}$ & $\phi$ & Crystal & $\beta F$ & $\mu$ \\
\hline 0.95 & 400 & 0.7448 & $\mathcal{A} 1$ & 20.11 & 300.6 \\
& 400 & 0.7447 & $\mathcal{A} 2$ & 20.07 & 297.2 \\
\hline 0.85 & 300 & 0.7614 & $\mathcal{B} 1_{P}$ & 20.14 & 213.9 \\
& 300 & 0.7614 & $\mathcal{B} 2_{P}$ & 19.81 & 213.6 \\
\hline 0.80 & 100 & 0.7301 & $\mathcal{B} 1_{P}$ & 15.27 & 71.50 \\
& 100 & 0.7300 & $\mathcal{B} 2_{P}$ & 15.19 & 71.42 \\
\hline 0.75 & 300 & 0.7684 & $\mathcal{B} 1_{P}$ & 20.16 & 192.7 \\
& 300 & 0.7684 & $\mathcal{B} 2_{P}$ & 20.14 & 192.6 \\
\hline 0.70 & 100 & 0.7240 & $\mathcal{B} 1_{P}$ & 14.80 & 71.50 \\
& 100 & 0.7243 & $\mathcal{B} 2_{P}$ & 14.74 & 71.42 \\
\hline 0.65 & 100 & 0.7143 & $\mathcal{B} 1_{P}$ & 14.54 & 67.19 \\
& 100 & 0.7141 & $\mathcal{B} 2_{P}$ & 14.46 & 67.12 \\
\hline 0.60 & 130 & 0.7325 & $\mathcal{C} 1$ & 16.29 & 62.75 \\
& 130 & 0.7325 & $\mathcal{C} 2$ & 16.06 & 62.51 \\
\hline 0.50 & 130 & 0.7220 & $\mathcal{C} 1$ & 16.32 & 63.46 \\
& 130 & 0.7220 & $\mathcal{C} 2$ & 16.19 & 63.34 \\
\hline 0.40 & 130 & 0.6596 & $\mathcal{C} 1$ & 14.18 & 42.13 \\
& 130 & 0.6605 & $\mathcal{C} 2$ & 14.00 & 41.90 \\
\hline 0.33 & 120 & 0.6444 & $\mathcal{C} 1$ & 14.48 & 39.33 \\
& 120 & 0.6443 & $\mathcal{C} 2$ & 14.51 & 39.36 \\
\hline 0.25 & 600 & 0.7401 & $\mathcal{C} 1$ & 20.406 & 86.772 \\
& 600 & 0.7401 & $\mathcal{C} 2$ & 20.407 & 86.774 \\
\hline & & & & & \\
\hline
\end{tabular}

Table 1 Results for the free energies of crystal candidates of CSCs with different values of $\chi^{*}$ that have similar structural and thermodynamic properties. Stable crystal structures are highlighted in grey.

\begin{tabular}{lccccc}
\hline$\chi^{*}$ & $P^{*}=P \sigma^{3}$ & $\phi$ & Crystal & $\beta F / N$ & $\mu$ \\
\hline 0.95 & 731 & 0.7310 & $\mathcal{P C}$ & 17.1361 & 277.008 \\
& 731 & 0.7696 & $\mathcal{A} 1$ & 22.4994 & 276.520 \\
& 731 & 0.7496 & $\mathcal{A} 2$ & 22.4990 & 276.519 \\
& 731 & 0.7503 & $\mathcal{B} 1_{P}$ & 23.6741 & 277.289 \\
& 731 & 0.7503 & $\mathcal{B} 2_{P}$ & 23.2761 & 276.887 \\
\hline 0.85 & 76.6 & 0.6656 & $\mathcal{P C}$ & 10.2193 & 66.8169 \\
& 76.6 & 0.7099 & $\mathcal{A} 1$ & 13.3603 & 66.4308 \\
& 76.6 & 0.7099 & $\mathcal{A} 2$ & 13.2444 & 66.3149 \\
& 76.6 & 0.7115 & $\mathcal{B} 1_{P}$ & 13.8182 & 66.7414 \\
& 76.6 & 0.7116 & $\mathcal{B} 2_{P}$ & 13.3894 & 66.4763 \\
\hline 0.75 & 49.7 & 0.6218 & $\mathcal{P C}$ & 9.3446 & 44.6377 \\
& 49.7 & 0.6648 & $\mathcal{A} 1$ & 11.4337 & 44.4559 \\
& 49.7 & 0.6649 & $\mathcal{A} 2$ & 11.3106 & 44.3202 \\
& 49.7 & 0.6766 & $\mathcal{B} 1_{P}$ & 11.7842 & 44.2168 \\
& 49.7 & 0.6770 & $\mathcal{B} 2_{P}$ & 11.7070 & 44.1519 \\
\hline
\end{tabular}

Table 2 Results for the free energies of crystal candidates of CSCs with different values of $\chi^{*}$ that have different structural and thermodynamic properties. Results are reported for the value of $P^{*}$ which corresponds to a $\mathcal{P C}$ at a supersaturation of $\Delta \mu^{*} \approx 0.5$. Stable crystal structures are highlighted in grey.

candidates are shown in Tables 1 and 2. It is evident from the results in Table 1 that crystal candidates with similar properties have small differences in the free energy. It must be noted that 


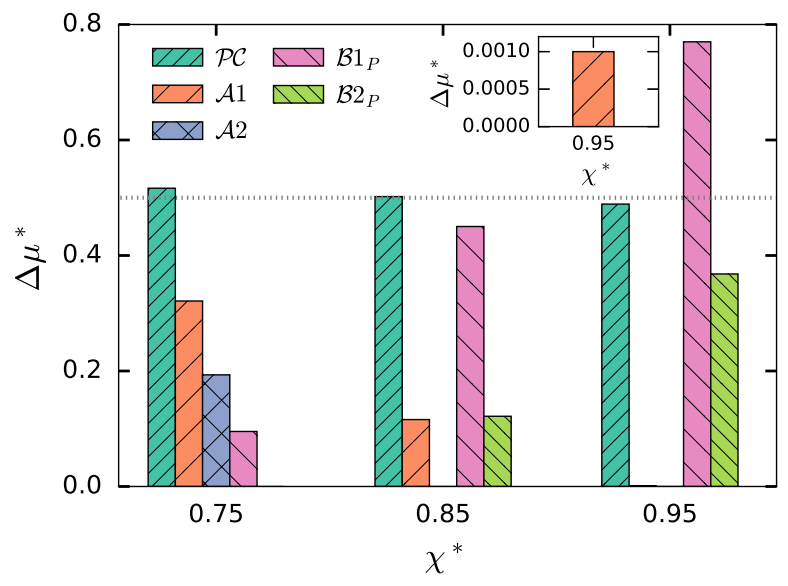

Fig. 4 Difference in chemical potential, $\Delta \mu^{*}$, between each metastable crystal and the stable crystal for different values of $\chi^{*} . \Delta \mu^{*}$ is measured at a $P^{*}$ which corresponds to a supersaturated $\mathcal{P} C$ with $\Delta \mu^{*} \approx 0.5$; the dotted line is shown at $\Delta \mu^{*}=0.5$ for clarity. The stable crystals at each aspect ratio are: $\chi^{*}=0.75-\mathcal{B} 2 ; \chi^{*}=0.85-\mathcal{A} 2 ; \chi^{*}=0.95-\mathcal{A} 2$. See Table 2 for more details.

given such small differences, such as for $\mathcal{C} 1$ and $\mathcal{C} 2$ at $\chi^{*}=0.25$ and for $\mathcal{A} 1$ and $\mathcal{A} 2$ at $\chi^{*}=0.95$, judgements on which phase is the most stable requires further analysis as the precision of our calculation is not high enough. For this reason, only approximate boundaries are drawn in the phase diagram shown in Figure 5. Relative stability may be calculated more accurately using, for example, the Monte Carlo lattice-switch method ${ }^{90}$. Such analysis, however, is outside the scope of the present work. As a contrast, Table 2 shows clear differences in the free energy of crystals with distinct structures. However, the stability at a given pressure is not determined by the free energy alone, but rather by the chemical potential. Examining the chemical potential of the $\mathcal{P} C, \mathcal{B}$ and $\mathcal{A}$ phases for $\chi^{*} \gtrsim 0.7$ reveals that while only one phase is stable at any time, many phases are closely metastable. In order to study the regions of metastability we compressed the $\mathcal{P} C$ phase until the system is metastable with a supersaturation of $\Delta \mu^{*} \approx 0.5$. At this pressure we report the difference in chemical potential, $\Delta \mu^{*}$, between the stable state - which depends on the aspect ratio and the various metastable states. The results are shown in both Figure 4 and Table 2, where it is evident from the small differences in chemical potential that a $\mathcal{P} C$ compressed past the point of stability will not necessarily transition into a single crystal type.

A summary of phase transitions for each value of $\chi^{*}$ studied is given in Table 3 and illustrated in a phase diagram in Figure 5. Particles with $\chi^{*}>0.95$ essentially crystallise in a similar way as hard spheres at moderate values of volume fraction. The results for the phase behaviour of CSCs with $\chi^{*}=0.95$ are shown in Figure 6. This system exhibits two main phase transitions: (a) transition from the $\mathcal{I}$ phase $(\phi=0.4922)$ to the $\mathcal{P} C$ phase $(\phi=0.5420)$ at $P^{*}=11.79$; and (b) transition from the $\mathcal{P} C$ phase $(\phi=0.7304)$ to the $\mathcal{A} 2$ phase $(\phi=0.7488)$ at $P^{*}=636.4$. The final phase transition to the $\mathcal{B} 2$ phase is of minor importance as it occurs at approximately $99.6 \%$ of $\phi_{m}$.

Figure 5 shows that $\mathcal{P} C$ is stable until about $\chi^{*}=0.74$. At $\chi^{*}=0.75$ there is a point where the density of the metastable fluid

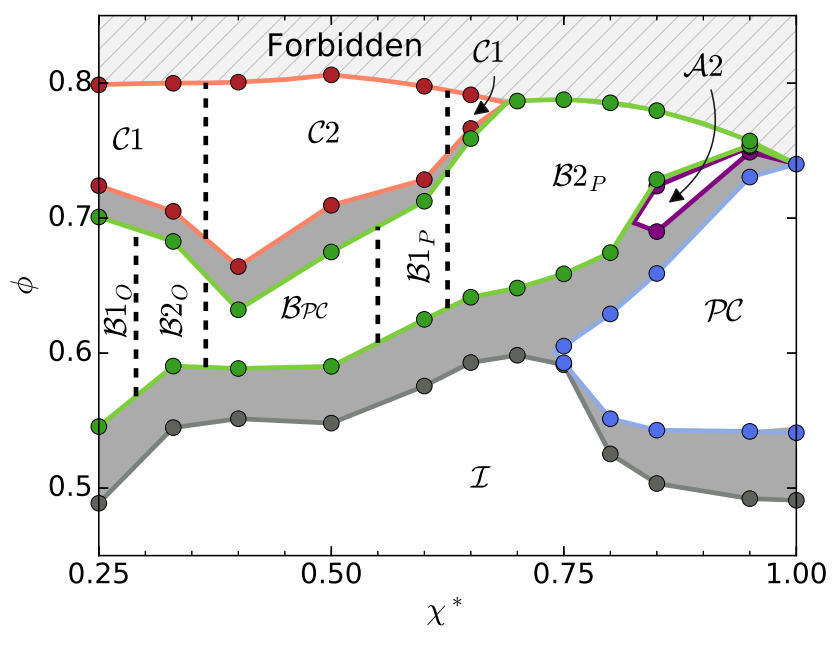

Fig. 5 Approximate aspect ratio-volume fraction $\left(\chi^{*}-\phi\right)$ phase diagram for CSCs. Circles represent results obtained from free energy calculations and NPT Monte Carlo simulations. The grey area represents coexistence regions, while the hatched area at the top of the phase diagram indicates the inaccessible densities where maximum packing fractions would be exceeded. Lines connecting the simulation points are used to guide the eye. Dotted lines denote approximate phase boundaries.

is greater than the density of the $\mathcal{P} C$. This suggests that there may be a point on the phase diagram where there is an isochoric, first order $\mathcal{I}$-P $C$ transition. $\mathcal{A} 2$ is the next stable phase observed across the range $0.95 \gtrsim \chi^{*} \gtrsim 0.85$, and the region of metastability extends to $\chi^{*}=0.7$ as is partly shown in Figure 4. $\mathcal{A} 1$ is not found to be the most stable phase at any point, but it is metastable across the range $0.95 \gtrsim \chi^{*} \gtrsim 0.70$. The region of maximum stability for $\mathcal{A} 2$ coincides with the maximum $\phi_{m}$ seen in Figure 3. It seems that the small increase in rotational freedom afforded by the $\mathcal{A}$ arrangement as compared to $\mathcal{B}$ is sufficient to offset the difference in packing efficiency between the two phases. However as the disparity in $\phi_{m}$ increases, $\mathcal{A}$ phases become metastable. For low anisotropy the $\mathcal{B}$ crystals are the most stable at high density, but at about $\chi^{*} \lesssim 0.689$ crystals of type $\mathcal{C}$ become the most dense stable crystals. Examining the range $0.6 \lesssim \chi^{*} \lesssim 0.69$, even though $\mathcal{C}$ crystals have greater $\phi_{m}, \mathcal{B}$ crystals exhibit much greater regions of stability, once more demonstrating the importance of rotational freedom. For $\chi^{*}=0.4$ one can make similar arguments for the stability of the $\mathcal{B}_{\mathcal{P C}}$ phase as were made previously for the $\mathcal{P C}$ phase: if we assume that the $\mathcal{B}_{\mathcal{P C}}$ phase maintains full rotational freedom then the theoretical maximum packing at $\chi^{*}=0.4$ must be significantly reduced. However we find that orientational order increases with packing fraction, indicating that the $\mathcal{B}_{\mathcal{P C}}$ crystal unit cell does deform and the dimers experience a reduction of rotational freedom. Thus the small region of stability for the $\mathcal{B}_{\mathcal{P C}}$ at this point is due partly to both reduced rotational freedom and inefficient packing. Finally, for similar crystals such as $\mathcal{A} 1$ and $\mathcal{A} 2$ it appears that the structures in which CSCs align along two principal axes are generally more stable. Determining exactly why this is so is inherently difficult, but it is suggested this is due to increased entropy as a result of particles being able to occupy twice as many orientations. 


\begin{tabular}{lllcccc}
\hline$\chi^{*}$ & \multicolumn{1}{l}{ Phase 1} & Phase 2 & $\phi_{1}$ & $\phi_{2}$ & $P^{*}$ & $\mu^{*}$ \\
\hline 0.99 & $\mathcal{I}$ & $\mathcal{P C}$ & 0.494 & 0.545 & 11.55 & 16.04 \\
\hline 0.95 & $\mathcal{I}$ & $\mathcal{P C}$ & 0.4922 & 0.5420 & 11.79 & 16.26 \\
& $\mathcal{P C}$ & $\mathcal{A} 2$ & 0.7304 & 0.7488 & 636.4 & 243.2 \\
& $\mathcal{A}$ & $\mathcal{B} 2_{P}$ & 0.7527 & 0.7539 & 1516 & 1072 \\
\hline 0.85 & $\mathcal{I}$ & $\mathcal{P C}$ & 0.5034 & 0.5429 & 14.02 & 18.00 \\
& $\mathcal{P C}$ & $\mathcal{A} 2$ & 0.6590 & 0.6900 & 65.34 & 58.46 \\
& $\mathcal{A} 2$ & $\mathcal{B} 2_{P}$ & 0.7237 & 0.7285 & 101.8 & 83.65 \\
\hline 0.80 & $\mathcal{I}$ & $\mathcal{P C}$ & 0.5253 & 0.5514 & 18.05 & 21.21 \\
& $\mathcal{P C}$ & $\mathcal{B} 2_{P}$ & 0.6290 & 0.6741 & 48.03 & 44.72 \\
\hline 0.75 & $\mathcal{I}$ & $\mathcal{P C}$ & 0.5914 & 0.5928 & 35.48 & 34.32 \\
0.75 & $\mathcal{P C}$ & $\mathcal{B} 2_{P}$ & 0.6052 & 0.6587 & 41.02 & 38.41 \\
\hline 0.70 & $\mathcal{I}$ & $\mathcal{B} 2_{P}$ & 0.5984 & 0.6482 & 41.56 & 36.96 \\
\hline 0.65 & $\mathcal{I}$ & $\mathcal{B} 2_{P}$ & 0.5930 & 0.6414 & 43.18 & 35.98 \\
& $\mathcal{B} 2_{P}$ & $\mathcal{C} 1$ & 0.7589 & 0.7664 & 324.6 & 180.6 \\
\hline 0.60 & $\mathcal{I}$ & $\mathcal{B} 1_{P}$ & 0.5756 & 0.6250 & 42.33 & 33.22 \\
& $\mathcal{B} 1_{P}$ & $\mathcal{C} 2$ & 0.7125 & 0.7285 & 122.3 & 73.06 \\
\hline 0.50 & $\mathcal{I}$ & $\mathcal{B}_{\mathcal{P C}}$ & 0.5481 & 0.5988 & 45.93 & 30.06 \\
& $\mathcal{B}_{\mathcal{P C}}$ & $\mathcal{C} 2$ & 0.6736 & 0.7076 & 108.3 & 55.40 \\
\hline 0.40 & $\mathcal{I}$ & $\mathcal{B}_{\mathcal{P C}}$ & 0.5514 & 0.5886 & 67.67 & 32.05 \\
& $\mathcal{B} \mathcal{P C}_{1}$ & $\mathcal{C} 2$ & 0.6321 & 0.6641 & 105.4 & 43.39 \\
\hline 0.33 & $\mathcal{I}$ & $\mathcal{B} 2_{O}$ & 0.5448 & 0.5904 & 89.97 & 32.21 \\
& $\mathcal{B} 2_{O}$ & $\mathcal{C} 2$ & 0.6827 & 0.7050 & 218.1 & 58.61 \\
\hline 0.25 & $\mathcal{I}$ & $\mathcal{B} 1_{O}$ & 0.4888 & 0.5456 & 100.4 & 26.23 \\
& $\mathcal{B} 1_{O}$ & $\mathcal{C} 1$ & 0.7010 & 0.7242 & 454.3 & 70.48 \\
\hline & & & & & & \\
\hline
\end{tabular}

Table 3 Crystal structures formed by CSC as function of the aspect ratio $\chi^{*}$. For each system, coexistence phases, coexistence volume fractions, transition pressure, and transition chemical potential $\mu^{*}$ are presented.

The results for the phase transitions are determined completely using free energy calculations to connect the equations of state of two stable phases in coexistence. However, it is important to stress that, with the exception of the $\mathcal{P} C$ phase for CSCs with large values of $\chi^{*}$, we do not observe the spontaneous formation of ordered phases from the compression of the isotropic phase. This is most likely due to a problem of slow kinetics. The formation of $\mathcal{B}$ phases, for example, requires as a first step the formation of dimer-like arrangements, followed by the organisation of the dimers into the final structure. This issue is exemplified in Figure 7 where the results for the equation of state and phase coexistence between the $\mathcal{I}$ phase $(\phi=0.5481)$ and the plastic crystal $\mathcal{B}_{\mathcal{P C}}$ phase $(\phi=0.5988)$ at $P^{*}=45.93$ formed by spherelike dimers of hemispheres $\left(\chi^{*}=0.5\right)$ are presented. The compression of the $\mathcal{I}$ phase pushes the system beyond the transition point reaching the supersaturated region. In the $\mathcal{B}_{\mathcal{P C}}$ phase all hemispheres need to be bonded through SEBs. The fraction of non-bonded hemispheres $f_{\text {unbonded }}$ as a function of the volume fraction is shown in Figure 7, where it is observed that at the entrance of the coexistence region, only $20 \%$ of hemispheres form dimers. Further compression of the system deep in the supersaturation region increases the fraction of bonded particles to $40 \%$, however, the acceptance of MC moves and the mobility of the sys-
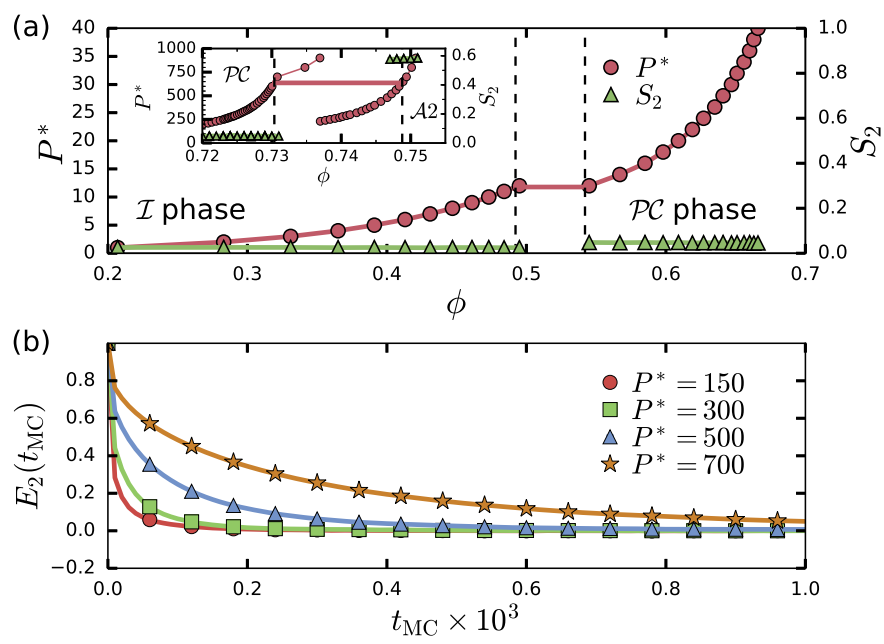

Fig. 6 Phase behaviour of CSCs with $\chi^{*}=0.95$. (a) Packing fraction-pressure $\left(\phi-P^{*}\right)$ equation of state and packing fraction-nematic order parameter $\left(\phi-S_{2}\right)$ profile obtained from NPT-MC simulations. (b) orientation autocorrelation function $E_{2}$ for pressures $P^{*}=[150,300,500,700]$ that correspond to states in the $\mathcal{P} C$ phase region. The maximum particle rotation is fixed at $\sim 26.5^{\circ}$.

tem characterised by the mean-square displacement (see Figure 7 (c)) are reduced significantly and the system is trapped in a disordered solid state. In another study, hard hemispheres were only found to crystallise when a significant concentration of depletants is added ${ }^{91}$. A similar trend is observed for the other transitions. For the system of CSCs with $\chi^{*}=0.95$, the system upon compression is able to transform from the $\mathcal{I}$ phase to the $\mathcal{P} C$ phase (see Figure 6), however, the system is unable to transform from the $\mathcal{P} C$ phase to an $\mathcal{A}$ phase. The formation of an $\mathcal{A}$ phase requires that all particles align along one or two directions which is characterised by both order parameters $S_{1}$ and $S_{2}$. As in the previous case of hemispheres, the system near the coexistence region becomes so dense that the acceptance of MC moves decreases significantly without an enhancement of the alignment of the particles as demonstrated by the behaviour of the orientation autocorrelation function $E_{2}$ shown in Figure 6(b). A more detailed analysis of the formation of ordered phases of CSCs in bulk requires a study of nucleation kinetics using special techniques for rare events. ${ }^{92-95}$

\subsection{Template-assisted self-assembly under gravity}

It is evident from the difficulty of forming ordered phases of CSCs in bulk that we require a different approach to promote the formation of different crystal phases. Directed self-assembly of CSCs has been studied using geometrical confinement ${ }^{56,58}$, and by using depletants to create an attractive interaction between hemispheres ${ }^{91}$. However, recent sedimentation experiments of particles with similar geometry as CSCs reveal the formation of ordered phases by stacking of layers on a planar surface ${ }^{60}$. In order to ascertain how CSCs self-assemble under gravity, we slowly decrease the gravitational length $l_{g}$ (increase of the gravitational strength) to observe the evolution of the stacking of layers and their structure first against a structureless hard wall. This could 

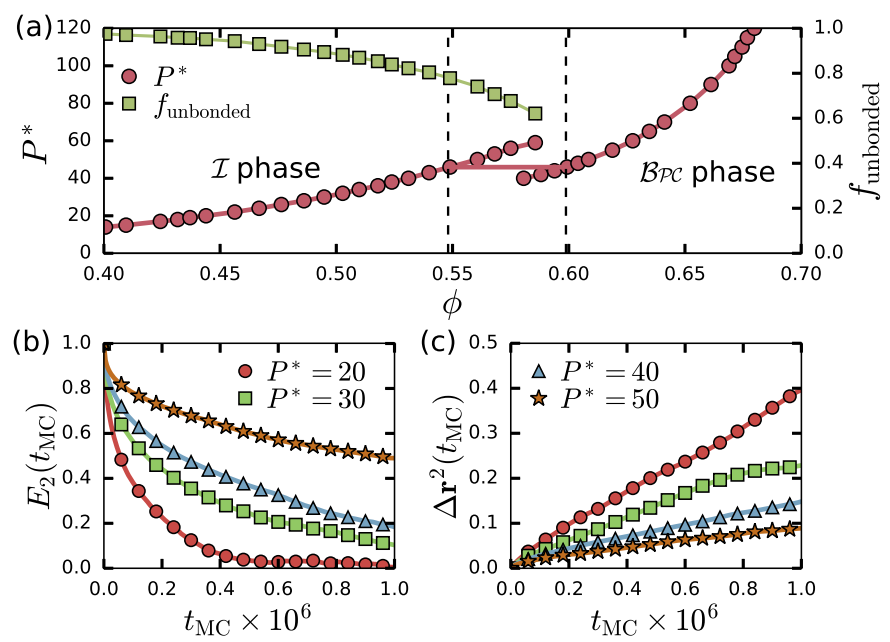

Fig. 7 Phase behaviour of CSCs with $\chi^{*}=0.5$. (a) Packing fraction-pressure $\left(\phi-P^{*}\right)$ equation of state obtained from NPT-MC simulations. The $\mathcal{I}$ phase branch is obtained from compression runs, while the crystal $\mathcal{B}_{\mathcal{P C}}$ branch is obtained from expansion of the crystal structure. Dashed lines indicate the point of the phase transition. The fraction of unpaired CSCs observed during the compression of the $\mathcal{I}$ phase is also shown. In (b) and (c) the orientation autocorrelation function $E_{2}$ and the mean square displacement $\Delta \mathbf{r}^{2}$ for two state points of the fluid branch corresponding to pressure $P^{*}=30$ (fluid state) and $P^{*}=50$ (supersaturated state) are shown. The maximum particle rotation is set as $\sim 2.86^{\circ}$; the maximum particle displacement is set as $0.01 \sigma$.

be achieved experimentally via slow sedimentation or steppedforce centrifugation in a density-matched medium ${ }^{96,97}$. We restrict our analysis to CSCs with aspect ratios $\chi^{*} \geq 0.75$. Hemispheres were also studied, but it was observed that the gravitational field did not aid the crystallisation of the particles.

The results for the density profile and order parameters profile for CSCs with $\chi^{*}=0.95$ are shown in Figure 8 at two different values of gravitational length $l_{g}^{*}$. For the gravitational length $l_{g}^{*}=0.2$ (Figure 8(a)) the system clearly forms layers that grow in the direction perpendicular to the flat wall. These layers are characterised by well-defined peaks in the density profile. The analysis of the order parameters $\Psi_{6}, S_{1}$ and $S_{2}$ in each layer reveals the formation of layers with hexagonal order (large values of $\Psi_{6}$ ) that lack orientational order with values of order parameters $S_{1} \sim 0$ and $S_{2} \sim 0$. This behaviour is an indication of the formation of a $\mathcal{P C}$ layer. At a lower gravitational length $\left(l_{g}^{*}=0.04\right.$, Figure 8(b)), the particles belonging to the three $\mathcal{P C}$ layers near the wall align in a common direction to form orientationally ordered crystal $(\mathcal{O O})$ layers having well-defined translational and orientational order that, in this case, resembles the $\mathcal{A}$ crystal observed in bulk simulations (see crystals $\mathcal{A} 1$ and $\mathcal{A} 2$ in Figure 2). Similar analysis for systems of CSCs with $\chi^{*}=0.85$ and $\chi^{*}=0.75$ reveals that in addition to the $\mathcal{A}$ phase observed at low values of $l_{g}^{*}$, in some cases the aligned particles of one layer are paired to the particles of an upper layer to form dimers via SEBs in an arrangement similar to $\mathcal{B}$ crystals. The results for the structural properties of the first three layers near the flat wall formed by CSCs with $\chi^{*}=0.85$ are shown in Figure 9, demonstrating the trans- (a)
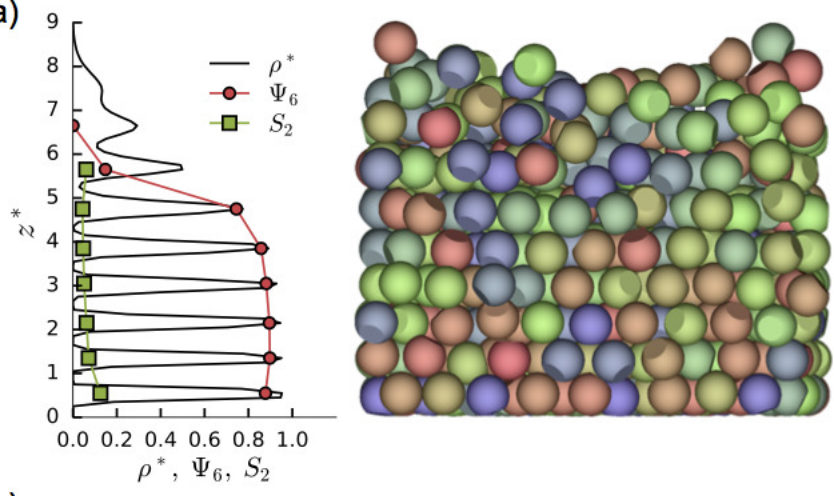

(b)

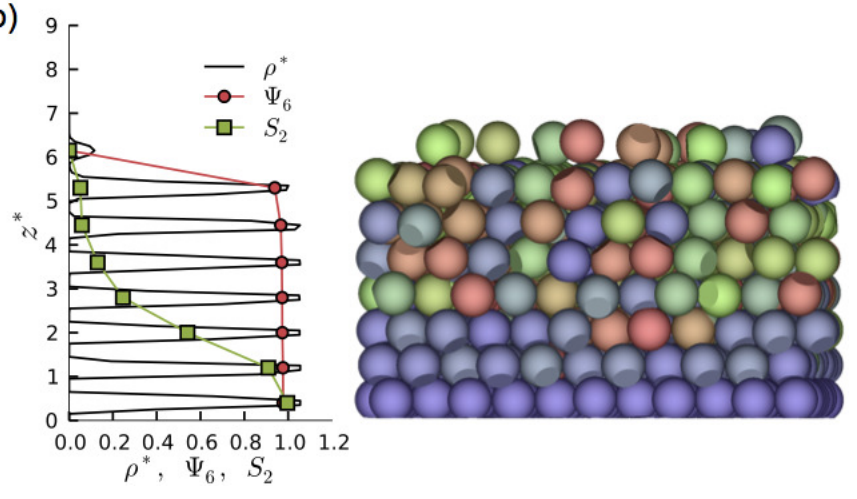

Fig. 8 Density $\rho^{*}$ and order parameters $\Psi_{6}$ and $S_{2}$ profiles along the direction of the gravity field for CSCs with $\chi^{*}=0.95$ for two values of gravitational length $l_{g}^{*}$. Representative configurations for each temperature are also shown. (a) Results for $l_{g}^{*}=0.20$ where the system exhibit coexistence of layers of $\mathcal{P C}$ structure with layers of $\mathcal{I}$ structure; (b) results for $l_{g}^{*}=0.04$. where three-phase coexistence of layers with $\mathcal{O O C}$ structure, $\mathcal{P C}$ structure, and $\mathcal{I}$ structure is observed.

formation of the layers from an $\mathcal{I}$ structure to a $\mathcal{P} C$ structure to an $\mathcal{O O C}$ structure upon increasing gravitational strength. Overall the use of a gravitational field and a flat substrate improved the self-assembly of CSCs. However, the methodology is limited as defects are increasingly common as the amount of anisotropy increases, and we observe crystals with different structures with slightly different input parameters. In other words, there is still an absence of control over the final outcome. In Figure 10, for example, two structures formed in systems of CSCs with $\chi^{*}=0.85$ are shown. Using different values for the area density, $\rho_{A}^{*}$, two different structures of the layers near the flat substrate are observed: (a) a herringbone stacking for $\rho_{A}^{*}=9$ and (b) a random stacking for $\rho_{A}^{*}=7.5$. This difficulty in controlling which structure is formed can be expected when the difference in chemical potential between crystals is so low as previously indicated in Figure 4 for bulk phases. A summary of the structures observed in systems of CSCs under gravity is presented in Table 4.

\subsubsection{Quantitative comparison: from bulk to gravity}

In addition to showing qualitatively the difference between bulk self-assembly and self-assembly under gravity we demonstrate quantitatively the effect gravity has on the phase behaviour of CSCs by calculating the pressure at which individual layers undergo phase transitions using Equation 9. We compare bulk coexistence pressures to the transition pressures observed under grav- 


\begin{tabular}{lcl}
\hline$\chi^{*}$ & Bulk Phase & Gravity \\
\hline 0.95 & $\mathcal{P C}$ & $\mathcal{P C} \mathcal{A} 1$ \\
0.85 & $\mathcal{P C}$ & $\mathcal{P C} \mathcal{A} 1 \mathcal{B} 1 \mathcal{B} 2$ \\
0.75 & & $\mathcal{P C} \mathcal{A} 1 \mathcal{B} 1$ \\
\hline
\end{tabular}

Table 4 Summary of ordered phases formed spontaneously in systems of CSCs with different values of $\chi^{*}$ under gravity. For comparison, the structures only obtained spontaneously by compression of the isotropic fluid in bulk conditions are also presented.

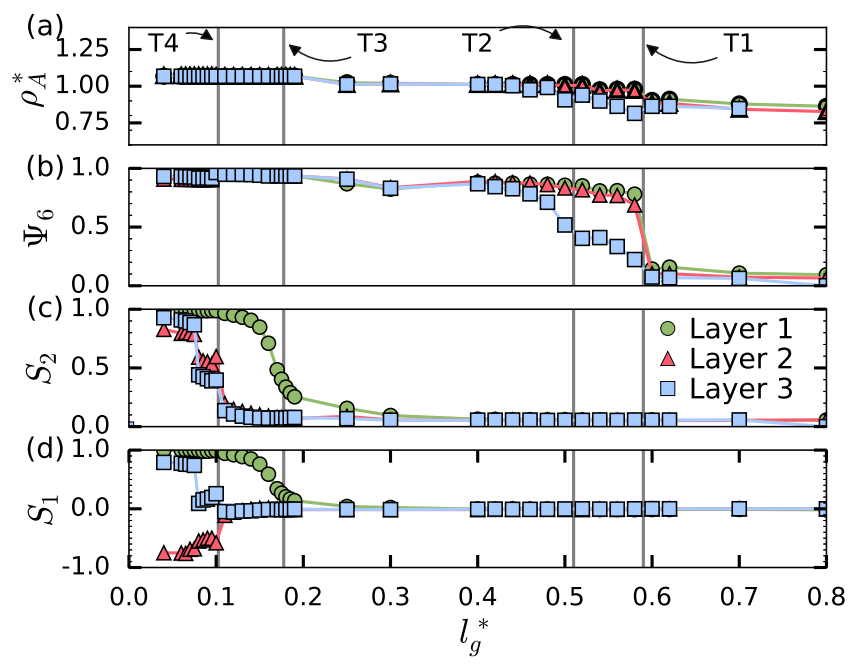

Fig. 9 Results for the structural properties of the layers formed by CSCs with $\chi^{*}=0.85$ The results correspond to (a) area density $\rho_{A}$, (b) hexagonal bond-order parameter $\Psi_{6}$, and orientational order parameters (c) $S_{2}$ and (d) $S_{1}$. Circles, triangles, and squares symbols correspond to the properties of the first, second, and third layers formed respectively. Vertical lines indicate the position of the phase transitions T1, T2, T3, and T4 in order of occurrence.

ity. Then in an attempt to pin-point the driving forces we also consider the strength of the gravitational field, and the influence of the structure of the substrate.

Since the only sequence of phase transitions observed in CSCs under gravity is $\mathcal{I}-\mathcal{P C}-\mathcal{O O C}$, there are two transitions to consider. First we examine the $\mathcal{P C}-\mathcal{I}$ transition. The results for the transition pressure $P^{*}$ and transition gravitational length $l_{g}^{*}$ as a function of the aspect ratio $\chi^{*}$ are shown in Figure 11 . The $\mathcal{P C}$ unit cell is almost identical to the FCC or HCP crystals formed by hard spheres, so it is useful to draw comparisons with previous studies on hard spheres. Hard spheres under gravity against a flat wall have been shown to crystallise at approximately the same pressure as bulk coexistence ${ }^{50}$. Hard spheres have also been studied without gravity at both flat walls and hexagonal templates ${ }^{79,98-101}$. These studies have shown that a flat wall induces hard spheres to crystallise at a slightly lower pressure than at bulk conditions, while a hexagonal template will significantly decrease the transition pressure. It can be seen in Figure 11 that CSCs mirror the behaviour of hard spheres: CSCs with $\chi^{*}=0.95$ under the influence of gravity exhibit a $\mathcal{P C}-\mathcal{I}$ transition at $P^{*}=11.8 \pm 0.1$, which is close to that of hard spheres in bulk ${ }^{102}\left(P_{\mathrm{hs}}^{*}=11.55\right)$ and
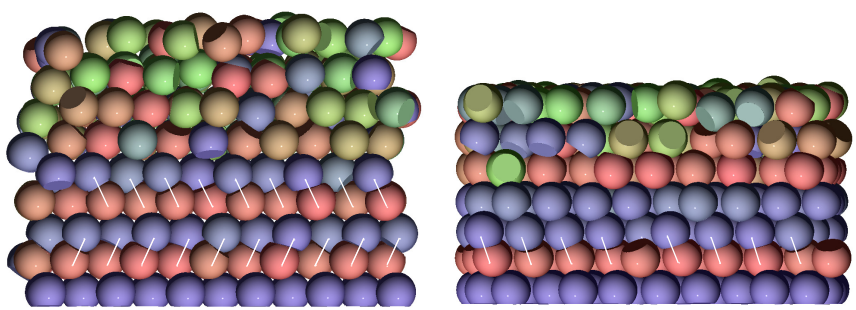

Fig. 10 Representative configuration of structures observed in systems of CSCs under gravity with different stacking orders. (a) herringbone stacking formed by CSCs with $\chi^{*}=0.85, \rho_{A}^{*}=9, l_{g}^{*}=0.04$; (b) random stacking formed by CSCs with $\chi^{*}=0.85, \rho_{A}^{*}=7.5, l_{g}^{*}=0.03$. Dimer bonds (SEBs) are indicated by white lines.
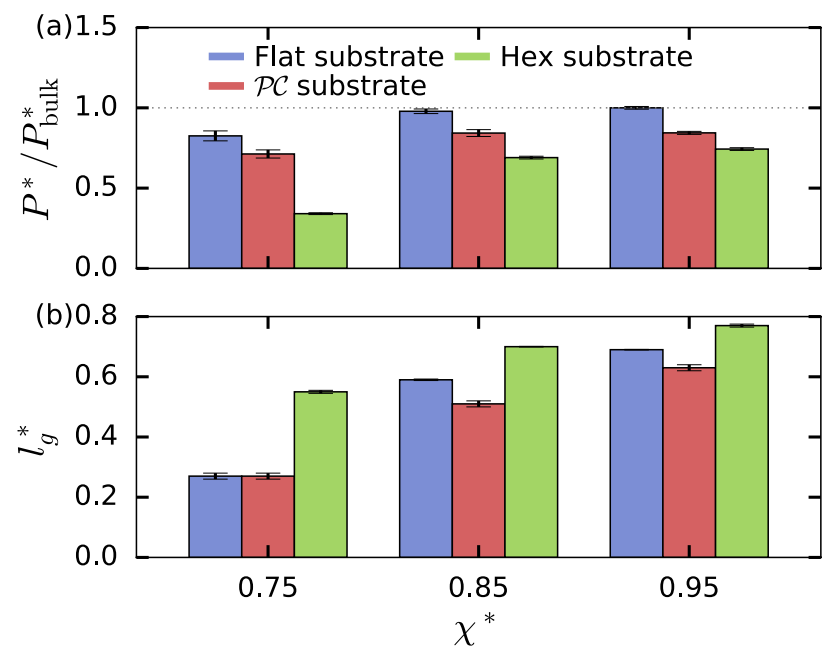

Fig. 11 Results for (a) the pressures $P^{*}$ and (b) gravitational length $l_{g}^{*}$ at the $\mathcal{I}$ - $\mathcal{P C}$ transition for CSCs under gravity. The transition pressures are presented relative to the transition pressures $P_{\text {bulk }}^{*}$ observed in bulk. The results correspond to (i) the formation of the first $\mathcal{P C}$ layer on top of the flat wall (denoted by 'Flat substrate'), (ii) the formation of a $\mathcal{P} C$ layer on top of a previously formed $\mathcal{P} C$ layer (denoted by ' $\mathcal{P} C$ substrate'), and (iii) the formation of the first $\mathcal{P} C$ layer on top of an artificially generated hexagonal layer of spheres (denoted by 'Hex substrate').

to that of CSCs with $\chi^{*}=0.95$ in bulk ( $P_{\mathrm{hs}}^{*}=11.79$ ); CSCs also form $\mathcal{P C}$ layers at significantly reduced pressures when in contact with a hexagonal template. A $\mathcal{P C}$ layer is also much more likely to crystallise when there is already a $\mathcal{P C}$ layer present, i.e. after the first layer of $\mathcal{P C}$ structure on the flat wall has formed. This first $\mathcal{P C}$ layer is effectively acting as a hexagonal template, albeit a defective one as the CSCs can rotate and have thermal motion. Along the same lines, it is evident that as the CSCs become more anisotropic the $\mathcal{P C}$ layer is decreasingly effective at promoting crystallisation when compared to a hexagonal template. It is then surprising that the flat wall has a much greater effect at stabilising $\mathcal{P C}$ layers for $\chi^{*}=0.75$, as there is no obvious entropic argument for this increased stability. This suggests that gravity also acts to stablise $\mathcal{P} C$ layers at pressures lower than bulk. This suggestion can also be inferred from Figure 11(b) which shows a correlation between the gravitational length and the transition pressure, noting that the gravitational length is inversely proportional to the gravitational strength. While such a strong effect has not been 
previously detected, it seems evident that particles should crystallise at pressures lower than bulk as more efficient packing leads to lower gravitational energy. This result highlights the need for a systematic investigation into the effect of gravitational strength on colloidal crystallisation.
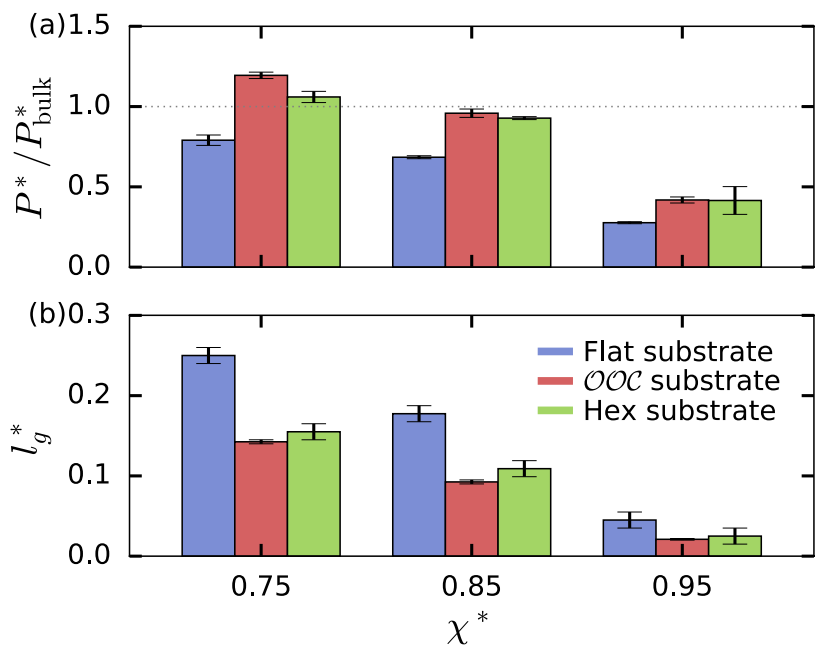

Fig. 12 Results for (a) the pressures $P^{*}$ and (b) gravitational length $l_{g}^{*}$ at the $\mathcal{P C}-\mathcal{O O C}$ transition for CSCs under gravity. The transition pressures are presented relative to the transition pressures $P_{\text {bulk }}^{*}$ observed in bulk. The results correspond to (i) the formation of the first $\mathcal{O O C}$ layer on top of the flat wall (denoted by 'Flat substrate'), (ii) the formation of an $\mathcal{O C C}$ layer on top of a previously formed $\mathcal{O C C}$ layer (denoted by ' $O C C$ substrate'), and (iii) the formation of the first $\mathcal{O O C}$ layer on top of an artificially generated hexagonal layer of spheres (denoted by 'Hex substrate').

Next we examine the $\mathcal{O O C}-\mathcal{P C}$ transition in Figure 12. Here we have compared the transition pressure under gravity with the coexistence pressure for whichever orientationally ordered phase is stable in bulk. The $\mathcal{O O C}$ label covers any type of orientationally ordered phase. This does not result in the most exact of comparisons, but considering that the orientationally ordered crystals are all close in stability this approximation is reasonable. For this transition there is no previously published work to compare our results to, but instead one can make arguments for what to expect. By aligning the flat face of the CSC against the wall the excluded volume is reduced. This entropic effect ought to be more pronounced as $\chi^{*}$ decreases. Similarly, by aligning this way the particle's centre of mass is lowered, thus decreasing its gravitational energy. Back to Figure 12 we can see that the transition occurs at a much lower pressure in the presence of a flat wall as has been suggested. Surprisingly though there appears to be a much stronger effect for higher values of $\chi^{*}$. This discrepancy may be explained by also considering the effect of $l_{g}^{*}$. For $\chi^{*}=0.95$ the transition happens at a very high gravitational strength. The discrepancy is consistent regardless of which surface the transition takes place on, suggesting that for this transition gravity is the dominant driving force. It can also be seen in Figure 12 that an $\mathcal{O O C}$ layer is almost as effective as a hexagonal template in promoting the formation of an $\mathcal{O O C}$ layer, more so than when comparing how a $\mathcal{P C}$ layer and a hexagonal template promote the formation of a $\mathcal{P C}$ layer. For example, Figure 11(a) shows that for $\chi^{*}=0.85$ the $\mathcal{I}$ - $\mathcal{P C}$ transition occurs at $P^{*}=0.84 P_{\text {bulk }}^{*}$ against a $\mathcal{P C}$ layer and at $P^{*}=0.69 P_{\text {bulk }}^{*}$ against a hexagonal template; while Figure 12 (a) shows that the $\mathcal{P C}-\mathcal{O O C}$ transition occurs at $P^{*}=0.96 P_{\text {bulk }}^{*}$ against a $\mathcal{P} C$ layer and at $P^{*}=0.93 P_{\text {bulk }}^{*}$ against a hexagonal template. On one hand this may be due to the absence of rotational freedom in the $\mathcal{O O C}$ layer; while on the other hand, thermal motion is also supressed as the layer is sandwiched in-between an $\mathcal{O O C}$ layer and a $\mathcal{P C}$ layer. It is also interesting to note that a hexagonal template seems to destabilise $\mathcal{O O C}$ layers for CSCs with $\chi^{*}=0.75$. From the previous results showing how gravity and a template will stabilise $\mathcal{P C}$ layers, it could be inferred that the increased stability of the $\mathcal{P C}$ layer is what is causing the $\mathcal{O O C}$ layer to have reduced stability. The results from Figures 11 and 12 are summarised in Table 5.

\begin{tabular}{llllll}
\hline \multirow{2}{*}{$\chi^{*}$} & Substrate & \multicolumn{2}{c}{ Transition $P^{*}$} & \multicolumn{2}{c}{ Transition $l_{g}^{*}$} \\
& & $\mathcal{I}-\mathcal{P C}$ & $\mathcal{P C}-\mathcal{O C}$ & $\mathcal{I}-\mathcal{P C}$ & $\mathcal{P C}-\mathcal{O C}$ \\
\hline 0.95 flat & $11.8(01)$ & $176(04)$ & $0.69(01)$ & $0.045(01)$ \\
& hex & $8.77(10)$ & $264(55)$ & $0.77(01)$ & $0.025(05)$ \\
$\mathcal{P C}$ & $9.96(11)$ & $\mathrm{N} / \mathrm{A}$ & $0.63(01)$ & $\mathrm{N} / \mathrm{A}$ \\
& $\mathcal{O} \mathcal{O C}$ & $\mathrm{N} / \mathrm{A}$ & $266(12)$ & $\mathrm{N} / \mathrm{A}$ & $0.021(01)$ \\
\hline 0.85 flat & $13.7(02)$ & $44.7(06)$ & $0.59(01)$ & $0.1775(25)$ \\
& hex & $9.66(12)$ & $60.6(06)$ & $0.70(01)$ & $0.109(01)$ \\
$\mathcal{P C}$ & $11.8(03)$ & $\mathrm{N} / \mathrm{A}$ & $0.51(01)$ & $\mathrm{N} / \mathrm{A}$ \\
& $\mathcal{O O C}$ & $\mathrm{N} / \mathrm{A}$ & $62.6(17)$ & $\mathrm{N} / \mathrm{A}$ & $0.0925(25)$ \\
\hline 0.75 flat & $29.3(11)$ & $31.7(13)$ & $0.27(01)$ & $0.25(01)$ \\
hex & $12.1(02)$ & $42.5(14)$ & $0.55(01)$ & $0.155(05)$ \\
$\mathcal{P C}$ & $25.3(09)$ & $\mathrm{N} / \mathrm{A}$ & $0.27(01)$ & $\mathrm{N} / \mathrm{A}$ \\
$\mathcal{O} \mathcal{O C}$ & N/A & $47.9(08)$ & N/A & $0.1425(25)$ \\
\hline
\end{tabular}

Table 5 Summary of the structures formed by CSCs under gravity. Results for the pressure $P^{*}$ and $l_{g}^{*}$ at the $\mathcal{P C}-\mathcal{I}$ and $\mathcal{O O C}-\mathcal{P C}$ layering transitions are also tabulated. Errors in the last two significant figures are indicated in the brackets.

\subsubsection{Nature of the phase transitions}

In addition to identifying the transitions exhibited by CSCs under gravity, we have also analysed the nature of the phase transitions of the layers. First we refer to previous experimental work on the phase transitions of hard spheres under gravity against a flat wall and a hexagonal template ${ }^{103}$. Hard spheres will undergo a continuous layerwise transition when in contact with a hexagonal template. Against a flat wall however the first two layers simultaneously undergo a first order phase transition. Here we are interested to see how introducing particle anisotropy will affect this behaviour, and whether this can shine some light on phenomena which are not well understood. Our results corroborate previous findings observed in hard spheres, but also reveal an additional example of a first order phase transition unique to anisotropic particles. In this work we identify first order phase transitions by discontinuous changes in order parameters, and the appearance of hysteresis; conversely second order transitions are marked by continuous changes in order parameters and a lack of hysteresis. A more comprehensive analysis of the order of phase transitions is outside the scope of this paper.

First and second order phase transitions are observed for both 


\begin{tabular}{lccll}
\hline Substrate & phase 1 & phase 2 & order & $n$ layers \\
\hline flat & $\mathcal{I}$ & $\mathcal{P C}$ & 1st order & multiple \\
& $\mathcal{P C}$ & $\mathcal{O O C}$ & 2nd order & single \\
\hline hex & $\mathcal{I}$ & $\mathcal{P C}$ & 2nd order & single \\
& $\mathcal{P C}$ & $\mathcal{O O C}$ & 2nd order & single \\
& $\mathcal{P C}$ & $\mathcal{O O C}$ & 1st order & multiple \\
\hline $\mathcal{P C}$ & $\mathcal{I}$ & $\mathcal{P C}$ & 2nd order & single \\
\hline $\mathcal{O O C}$ & $\mathcal{P C}$ & $\mathcal{O O C}$ & 2nd order & single \\
& $\mathcal{P C}$ & $\mathcal{O O C}$ & 1st order & multiple \\
\hline
\end{tabular}

Table 6 Overview of the different phase transitions of CSC layers under gravity. The surface or layer upon which the transition takes place is indicated, along with the order of the transition and whether a single layer is involved or multiple layers simultaneously undergo a phase transition.

the $\mathcal{I}-\mathcal{P C}$ and the $\mathcal{P C}-\mathcal{O} \mathcal{O C}$ transformation. Figure 9 shows the order parameters for the first three layers of CSCs for $\chi^{*}=0.85$ against a flat wall, with phase transitions indicated by $\mathrm{T} 1$ to $\mathrm{T} 4$. At T1 the first two layers undergo a first order $\mathcal{I}$ - $\mathcal{P C}$ phase transition against a flat wall. At T2 the third layer undergoes a second order $\mathcal{I}-\mathcal{P C}$ phase transition while in contact with a $\mathcal{P C}$ layer, which is the same type of transition one sees for CSCs in contact with a hexagonal template (results not shown). At T3 the first layer undergoes a second order $\mathcal{P C}-\mathcal{O O C}$ transition against a flat wall. Finally, at T4 the second and third layers simultaneously undergo a first order $\mathcal{P C}-\mathcal{O} \mathcal{O C}$ phase transition. This final example, to our knowledge, is a new phenomenon where two layers simultaneously transform as CSCs from one layer form dimers with CSCs from the other layer in a cooperative event. Though not explicitly shown, hysteresis is only observed for transitions with discontinuous changes in order parameters.

A summary of all the phase transitions observed in this work is shown in table 6 . First order phase transitions are observed for fluid-crystal transitions at a flat wall, and for transitions where two layers form dimers between them. It is clear why two layers crystallise simultaneously to form dimers as this is a cooperative event, though it is not evident why two layers of CSCs or hard spheres crystallise simultaneously at a flat wall. In the case of the flat wall, as discussed in the previous section, CSCs will crystallise against a flat wall at a pressure close to that of coexistence in bulk, while they will crystallise against a $\mathcal{P C}$ layer at a lower pressure. The first layer to crystallise acts to an extent like a hexagonal template and enables the formation of the second layer at the same time. Second order transitions appear to occur when particles can individually make a transition from one state to the other without otherwise disturbing the local structure, such as: a single particle aligning up-down in a $\mathcal{P C}$ layer; or a single particle occupying a lattice site in a hexagonal template. This results in transitions where the equilibrium concentration of particles in one state or the other can smoothly vary. Conversely, first order phase transitions appear as a feature of emergent behaviour, where particles move co-operatively.

\section{Conclusions}

In conclusion, we have mapped the phase diagram for convex spherical caps with aspect ratio $\chi^{*}$ over the range $0.25 \leq \chi^{*} \leq 1.0$. Our results contribute to demonstrate the strong effect of changes of shape anisotropy in non-centrosymmetric CSC particles on the complexity of the ordered structures. Rather like how hard spheres may form FCC, HCP or random HCP crystals, CSCs also exhibit configurational degeneracy whereby at a given state point several crystal phases may exist separated by small differences in chemical potential. At intermediate densities CSCs prefer configurations where they have rotational freedom, such as plastic crystals; while at high densities they prefer close-packed structures, indicating the competition between packing entropy and rotational entropy as driving forces. The phase transitions of CSCs in bulk occur at densities and pressures higher than that of hard spheres. At these densities CSCs are less mobile and rotational freedom is constrained which frustrates crystallisation. This effect is more pronounced as shape anisotropy is increased, resulting in only a small part of the phase diagram being accessible via spontaneous self-assembly from compression of the isotropic phase. The additional existence of closely metastable states as indicated by free energy calculations suggests that it is difficult to control the nucleation of a single type of crystal. We further investigated the self-assembly of convex spherical caps driven by the sedimentation of the particles onto different substrates. We have observed an enhancement of the spontaneous formation of other crystals beyond the $\mathcal{P} C$ phase, but it is still difficult to control the final crystal structure. Moreover, we observed that a gravitational field does not help crystallisation of CSCs with high anisotropy. Nonetheless, the reasons for the improved self-assembly that we observe may be threefold. First, a template, whether it be a flat wall, a hexagonal template or a crystal surface, will help the system to overcome the nucleation free-energy barrier. Second, gravity and a template act together to increase the stability of the crystal structures so they crystallise at lower pressures; this corresponds to crystallising at lower densities where particles have increased mobility. And third, it is suggested that phase transitions for hard particles at high densities may favour second order phase transitions like epitaxial growth on a surface ${ }^{66}$. To further understand the crystallisation of these systems, analysis of nucleation kinetics is required.

\section{Acknowledgements}

The authors thank Dr. Matthieu Marechal of Friedrich-Alexander University Erlangen-Nürnberg and Dr. Ran Ni of Nanyang Technological University for discussions pertaining to the phase behaviour of hard hemispheres. We thank the Computational Shared Facility of the University of Manchester for computing time. This work acknlowledges Doctoral Training Award funding from the University of Manchester.

\section{A Free Energy}

Free energy calculations are used in this work to pinpoint the location of phase transitions, and to determine the relative stability of crystals. The free energy $F(\rho)$ of a system at density $\rho$ is cal- 
culated by integration of the equation of state from a reference state of density $\rho_{0}$ and free energy $F\left(\rho_{0}\right)$ as

$$
\frac{\beta}{N} F(\rho)=\frac{\beta}{N} F\left(\rho_{0}\right)+\int_{\rho_{0}}^{\rho} \mathrm{d} \rho^{\prime}\left(\frac{\beta P\left(\rho^{\prime}\right)}{\rho^{\prime 2}}\right),
$$

where $\beta=1 /\left(k_{B} T\right)$. Equation 10 is only valid if the system does not cross a first order phase transition. For fluids the free energy of the reference state is obtained using the Euler equation, $F\left(\rho_{0}\right) / N=\mu\left(\rho_{0}\right)-P\left(\rho_{0}\right) / \rho_{0}$, where the chemical potential at density $\rho_{0}$ is obtained using the Widom particle insertion $\operatorname{method}^{70,71}$. For solids the free energy, $F\left(\rho_{0}\right)$ is obtained using the Einstein crystal method $68,69,104,104$. The Einstein crystal method involves constructing a reversible thermodynamic path from the real solid to a non-interacting harmonic crystal. For the case of an orientationally ordered crystal $(\mathcal{O O C})$, this is achieved by coupling both positions and orientations to the lattice positions using the potential energy functions $U_{\text {Ein, } O O C C}$ given by

$$
U_{\text {Ein, } \mathcal{O O C}}=\sum_{i<j} \phi_{i j}^{h b}+\zeta\left[\lambda_{r} \sum_{i=1}^{N}\left(r_{i}-r_{0, i}\right)^{2}+\lambda_{u} \sum_{i=1}^{N}\left(1-\cos \left(\theta_{i 0}\right)\right]\right.
$$

where $\phi_{i j}^{h b}$ is the hard-core interaction, $\lambda_{r}$ and $\lambda_{u}$ are the constants coupling the particles to their lattice positions and orientations, respectively, $\zeta$ is the parameter turning the springs on or off, i.e. $0 \leq \zeta \leq 1, \mathbf{r}_{0, i}$ is the lattice position of particle $i, \theta_{i 0}$ is the angle between the orientation of particle $i$ and its lattice orientation. The choice of potential for constraining orientations is dependent on the geometry of the particle. The values of $\lambda_{r}$ and $\lambda_{u}$ are chosen such that the particles do not interact at $\zeta=1$. In practice the system is considered non-interacting if the probability of particles overlapping is less than $0.01 \%$, which is achieved using values of up to $\lambda_{r}=\lambda_{u}=10^{6}$. The reference free energy $F_{\text {Ein, } \mathcal{O O C}}$ of an orientationally ordered Einstein crystal is given by

$$
\begin{aligned}
\frac{\beta}{N} F_{\operatorname{Ein}, \mathcal{O O C}}=-\frac{3(N-1)}{2 N} & \ln \left(\frac{\pi}{\beta \lambda_{r}}\right)-\ln \left(\frac{1-e^{-2 \lambda_{u}}}{\lambda_{u}}\right) \\
+ & \ln \left(\frac{\Lambda}{\sigma^{3}}\right)+\ln \left(\frac{\sigma^{3}}{V N^{1 / 2}}\right)+\ln (\mathcal{V})
\end{aligned}
$$

where $\Lambda$ is the thermal de Broglie wavelength, and $\mathcal{V}=$ $\left(h^{2} \beta / 2 \pi I\right)$, where $I$ is the moment of inertia. The free energy of the real solid $F(\rho)$ is calculated using thermodynamic integration

$$
\beta F(\rho)=\beta F_{\operatorname{Ein}, \mathcal{O O C}}-\int_{0}^{1} d \zeta\left\langle\frac{\partial U_{\operatorname{Ein}, \mathcal{O O C}}\left(\zeta, \lambda_{r}, \lambda_{u}\right)}{\partial \zeta}\right\rangle_{N, V, T, \zeta}
$$

Anisotropic particles which form plastic crystals will still interact when fixed to lattice positions due to their orientational freedom. To overcome this problem the hard potential is replaced with a penetrable step potential, $\phi_{i j}^{p e n}=\gamma A$. The interaction strength is controlled by $\gamma$, and $A$ is an arbitrary constant set to $A=0.1$. The choice of $A$ determines the $\gamma_{\max }$ at which the potential approximates a hard-core interaction. Thus $\phi_{i j}^{p e n}$ varies between non-interacting at $\gamma=0$ and a hard-core interaction at $\gamma_{\max }=250 .{ }^{32}$ The free energy of plastic crystals is then evaluated as:

$$
\begin{aligned}
\beta F(\rho)=\beta F_{\operatorname{Ein}, \mathcal{P C}} & -\int_{0}^{1} d \zeta\left\langle\frac{\partial U_{\operatorname{Ein}, \mathcal{P C}}\left(\zeta, \lambda_{r}, \gamma\right)}{\partial \zeta}\right\rangle_{N, V, T, \zeta, \gamma} \\
& +\int_{0}^{\gamma_{\max }} d \gamma\left\langle\frac{\partial U_{\operatorname{Ein}, \mathcal{P C}}\left(\zeta, \lambda_{r}, \gamma\right)}{\partial \gamma}\right\rangle_{N, V, T, \zeta, \gamma}
\end{aligned}
$$

where

$$
U_{\text {Ein }, \mathcal{P C}}\left(r^{N}, u^{N}, \lambda_{r}, \gamma\right)=\phi_{i j}^{p e n}+\zeta \lambda_{r} \sum_{i=1}^{N}\left(r_{i}-r_{0, i}\right)^{2}
$$

and

$$
\begin{array}{r}
\frac{\beta}{N} F_{\text {Ein }, \mathcal{P C}}=-\frac{3(N-1)}{2 N} \ln \left(\frac{\pi}{\beta \lambda_{r}}\right)+\ln \left(\frac{\Lambda}{\sigma^{3}}\right) \\
+\ln \left(\frac{\sigma^{3}}{V N^{1 / 2}}\right)+\ln (\mathcal{V})
\end{array}
$$

\section{References}

1 G. M. Whitesides and B. Grzybowski, Science, 2002, 295, 2418-2421.

2 B. A. Grzybowski, C. E. Wilmer, J. Kim, K. P. Browne and K. J. Bishop, Soft Matter, 2009, 5, 1110-1128.

3 A. Yethiraj and A. van Blaaderen, Nature, 2003, 421, 513517.

4 J.-W. Kim, R. J. Larsen and D. A. Weitz, J. Am. Chem. Soc., 2006, 128, 14374-14377.

5 S. Badaire, C. Cottin-Bizonne, J. W. Woody, A. Yang and A. D. Stroock, J. Am. Chem. Soc., 2007, 129, 40-41.

6 C. J. Hernandez and T. G. Mason, J. Phys. Chem. C, 2007, 111, 4477-4480.

7 S. Sacanna and D. J. Pine, Curr. Opin. Colloid Interface Sci., 2011, 16, 96-105.

8 S. Sacanna, M. Korpics, K. Rodriguez, L. Colón-Meléndez, S.-H. Kim, D. J. Pine and G.-R. Yi, Nat. Commun., 2013, 4, 1688.

9 M. Grzelczak, J. Vermant, E. M. Furst and L. M. Liz-Marzán, ACS Nano, 2010, 4, 3591-3605.

10 S. C. Glotzer and M. J. Solomon, Nat. Mater., 2007, 6, 557562.

11 L. Onsager, Ann. N. Y. Acad. Sci., 1949, 51, 627-659.

12 M. Hosino, H. Nakano and H. Kimura, J. Phys. Soc. Jpn., 1979, 46, 1709-1715.

13 M. Hosino, H. Nakano and H. Kimura, J. Phys. Soc. Jpn., 1979, 47, 740-745.

14 M. Hosino, H. Nakano and H. Kimura, J. Phys. Soc. Jpn., 1982, 51, 741-748.

15 D. Frenkel, J. Phys. Chem., 1988, 92, 3280-3284.

16 D. Frenkel, H. N. W. Lekkerkerker and A. Stroobants, Nature, 1988, 332, 822-823. 
17 S. C. McGrother, D. C. Williamson and G. Jackson, J. Chem. Phys., 1996, 104, 6755-6771.

18 P. Bolhuis and D. Frenkel, J. Chem. Phys., 1997, 106, 666687.

19 M. R. Jones, R. J. Macfarlane, B. Lee, J. Zhang, K. L. Young, A. J. Senesi and C. A. Mirkin, Nat. Mater., 2010, 9, 913-917.

20 D. Frenkel, Physica A, 1999, 263, 26-38.

21 F. A. Escobedo, Soft Matter, 2014, 10, 8388-8400.

22 G. van Anders, N. K. Ahmed, R. Smith, M. Engel and S. C. Glotzer, ACS Nano, 2013, 8, 931-940.

23 J. A. C. Veerman and D. Frenkel, Phys. Rev. A, 1992, 45, 5632-5648.

24 T. Schilling, S. Pronk, B. Mulder and D. Frenkel, Phys. Rev. E, 2005, 71, 036138.

25 C. Avendaño and F. A. Escobedo, Soft Matter, 2012, 8, 46754681.

26 S. P. Carmichael and M. S. Shell, J. Chem. Phys., 2013, 139, 164705.

27 X. Ye, J. Chen, M. Engel, J. A. Millan, W. Li, L. Qi, G. Xing, J. E. Collins, C. R. Kagan, J. Li et al., Nat. Chem., 2013, 5, 466-473.

28 J. A. Millan, D. Ortiz, G. van Anders and S. C. Glotzer, ACS Nano, 2014, 8, 2918-2928.

29 C. Vega, E. P. A. Paras and P. A. Monson, J. Chem. Phys., 1992, 96, 9060-9072.

30 K. Milinković, M. Dennison and M. Dijkstra, Phys. Rev. E, 2013, 87, 032128.

31 K. Muangnapoh, C. Avendaño, F. A. Escobedo and C. M. Liddell-Watson, Soft Matter, 2014, 10, 9729-9738.

32 M. Marechal and M. Dijkstra, Phys. Rev. E, 2010, 82, 031405.

33 M. Marechal, R. J. Kortschot, A. F. Demirörs, A. Imhof and M. Dijkstra, Nano Lett., 2010, 10, 1907-1911.

34 D. J. Ashton, R. L. Jack and N. B. Wilding, Soft Matter, 2013, 9, 9661-9666.

35 G. Cinacchi and J. S. van Duijneveldt, J. Phys. Chem. Lett., 2010, 1, 787-791.

36 G. Cinacchi, J. Chem. Phys., 2013, 139, 124908.

37 G. Cinacchi and A. Tani, J. Chem. Phys., 2014, 141, 154901.

38 S. Torquato and Y. Jiao, Nature, 2009, 460, 876-879.

39 S. Torquato and Y. Jiao, Phys. Rev. E, 2009, 80, 041104.

40 U. Agarwal and F. A. Escobedo, Nat. Mater., 2011, 10, 230235.

41 P. F. Damasceno, M. Engel and S. C. Glotzer, Science, 2012, 337, 453-457.

42 V. Thapar, T. Hanrath and F. A. Escobedo, Soft Matter, 2015, 11, 1481-1491.

43 J. de Graaf, R. van Roij and M. Dijkstra, Phys. Rev. Lett., 2011, 107, 155501.

44 S. Atkinson, Y. Jiao and S. Torquato, Phys. Rev. E, 2012, 86, 031302.

45 W. Qi, J. d. Graaf, F. Qiao, S. Marras, L. Manna and M. Dijkstra, Nano Lett., 2012, 12, 5299-5303.

46 M. P. Arciniegas, M. R. Kim, J. de Graaf, R. Brescia, S. Mar- ras, K. Miszta, M. Dijkstra, R. Van Roij and L. Manna, Nano Lett., 2014, 14, 1056-1063.

47 H. Löwen, J. Phys.: Condens. Matter, 2001, 13, R415.

48 S. V. Savenko and M. Dijkstra, Phys. Rev. E, 2004, 70, 051401.

49 M. Schmidt, M. Dijkstra and J.-P. Hansen, J. Phys.: Condens. Matter, 2004, 16, S4185.

50 M. Marechal and M. Dijkstra, Phys. Rev. E, 2007, 75, 061404.

51 M. Marechal, M. Hermes and M. Dijkstra, J. Chem. Phys., 2011, 135, 034510.

52 M. Marechal and M. Dijkstra, Soft Matter, 2011, 7, 13971408.

53 J. Henzie, M. Grünwald, A. Widmer-Cooper, P. L. Geissler and P. Yang, Nat. Mater., 2012, 11, 131-137.

54 J.-M. Meijer, D. V. Byelov, L. Rossi, A. Snigirev, I. Snigireva, A. P. Philipse and A. V. Petukhov, Soft Matter, 2013, 9, 10729-10738.

55 E. M. Furst, Soft Matter, 2013, 9, 9039-9045.

56 C. Avendaño, C. M. Liddell-Watson and F. A. Escobedo, Soft Matter, 2013, 9, 9153-9166.

57 I. D. Hosein and C. M. Liddell, Langmuir, 2007, 23, 88108814.

58 E. K. Riley and C. M. Liddell, Langmuir, 2010, 26, 11648 11656.

59 E. Y. K. Fung, K. Muangnapoh and C. M. Liddell Watson, J. Mater. Chem., 2012, 22, 10507-10513.

60 S. J. Ivell, R. P. A. Dullens, S. Sacanna and D. G. A. L. Aarts, Soft Matter, 2013, 9, 9361-9365.

61 S.-H. Kim, A. D. Hollingsworth, S. Sacanna, S.-J. Chang, G. Lee, D. J. Pine and G.-R. Yi, J. Am. Chem. Soc., 2012, 134, 16115-16118.

62 S. Torquato and Y. Jiao, Phys. Rev. E, 2012, 86, 011102.

63 E. Riley, E. Fung and C. M. Liddell-Watson, J. Appl. Phys., 2012, 111, 093504.

64 A. C. Stelson, C. Avendano and C. M. Liddell-Watson, J. Appl. Phys., 2016, 119, 023110.

65 A. C. Stelson, E. K. Riley and C. M. L. Watson, J. Opt. Soc. Am. B, 2016, 33, 1588-1593.

66 A. van Blaaderen, R. Ruel and P. Wiltzius, Nature, 1997, 385, 321-324.

67 R. Eppenga and D. Frenkel, Mol. Phys., 1984, 52, 13031334.

68 D. Frenkel and A. J. C. Ladd, J. Chem. Phys., 1984, 81, 31883193.

69 C. Vega, E. Sanz, J. L. F. Abascal and E. G. Noya, J. Phys. Condens. Matter, 2008, 20, 153101.

70 D. Frenkel and B. Smit, Understanding molecular simulation: from algorithms to applications, Academic press, 2001, vol. 1.

71 B. Widom, J. Chem. Phys., 1963, 39, 2808-2812.

72 R. Najafabadi and S. Yip, Scr. Metall., 1983, 17, 1199-1204.

73 L. Filion, M. Marechal, B. van Oorschot, D. Pelt, F. Smallenburg and M. Dijkstra, Phys. Rev. Lett., 2009, 103, 188302.

74 J. de Graaf, L. Filion, M. Marechal, R. van Roij and M. Dijk- 
stra, J. Chem. Phys., 2012, 137, 214101.

75 P. J. Steinhardt, D. R. Nelson and M. Ronchetti, Phys. Rev. Lett., 1981, 47, 1297-1300.

76 P. J. Steinhardt, D. R. Nelson and M. Ronchetti, Phys. Rev. B, 1983, 28, 784.

77 J. Vieillard-Baron, Mol. Phys., 1974, 28, 809-818.

78 A. Patti and A. Cuetos, Phys. Rev. E, 2012, 86, 011403.

79 S. Dorosz and T. Schilling, J. Chem. Phys., 2012, 136, 044702.

80 D. R. Nelson and B. Halperin, Phys. Rev. B, 1979, 19, 2457.

81 M. P. Allen and D. J. Tildesley, Computer simulation of liquids, Oxford university press, 1989.

82 T. Biben, R. Ohnesorge and H. Löwen, Europhys. Lett., 1994, 28, 665 .

83 G. van Anders, D. Klotsa, N. K. Ahmed, M. Engel and S. C. Glotzer, Proc. Natl. Acad. Sci. U.S.A., 2014, 111, E4812.

84 A. Donev, F. H. Stillinger, P. M. Chaikin and S. Torquato, Phys. Rev. Lett., 2004, 92, 255506.

85 P. Pfleiderer and T. Schilling, Phys. Rev. E, 2007, 75, 020402.

86 K. W. Wojciechowski, D. Frenkel and A. C. Brańka, Phys. Rev. Lett., 1991, 66, 3168-3171.

87 M. Marechal and M. Dijkstra, Phys. Rev. E, 2008, 77, 061405.

88 M. He and P. Siders, J. Phys. Chem., 1990, 94, 7280-7288.

89 G. Cinacchi and S. Torquato, J. Chem. Phys., 2015, 143, 224506.

90 A. D. Bruce, A. N. Jackson, G. J. Ackland and N. B. Wilding, Phys. Rev. E, 2000, 61, 906-919.

91 J. Glaser, A. S. Karas and S. C. Glotzer, J. Chem. Phys., 2015, 143, 184110.

92 S. Auer and D. Frenkel, Nature, 2001, 409, 1020-1023.

93 L. Filion, M. Hermes, R. Ni and M. Dijkstra, J. Chem. Phys., 2010, 133, 244115.

94 S. L. Meadley and F. A. Escobedo, J. Chem. Phys., 2012, 137, 074109.

95 V. Thapar and F. A. Escobedo, Phys. Rev. Lett., 2014, 112, 048301.

96 R. Piazza, T. Bellini and V. Degiorgio, Phys. Rev. Lett., 1993, 71, 4267-4270.

97 D. Mukhija and M. J. Solomon, Soft Matter, 2011, 7, 540545.

98 D. J. Courtemanche, T. A. Pasmore and F. Van Swol, Mol. Phys., 1993, 80, 861-875.

99 M. Dijkstra, Phys. Rev. Lett., 2004, 93, 108303.

100 M. Heni and H. Löwen, J. Phys. Condens. Matter, 2001, 13, 4675.

101 A. Cacciuto and D. Frenkel, Phys. Rev. E, 2005, 72, 041604.

102 E. G. Noya, C. Vega and E. de Miguel, J. Chem. Phys., 2008, 128, 154507.

103 I. B. Ramsteiner, K. E. Jensen, D. A. Weitz and F. Spaepen, Phys. Rev. E, 2009, 79, 011403.

104 J. M. Polson, E. Trizac, S. Pronk and D. Frenkel, J. Chem. Phys., 2000, 112, 5339-5342. 